\title{
Effects of self-attraction and loading at a regional scale: a test case for the Northwest European Shelf
}

\author{
Maialen Irazoqui Apecechea ${ }^{1}$ (D) Martin Verlaan ${ }^{1,2} \cdot$ Firmijn Zijl $^{1}$ - Camille Le Coz $^{2}$ • \\ Herman Kernkamp ${ }^{1}$
}

Received: 30 October 2016 / Accepted: 30 March 2017 /Published online: 4 May 2017

(C) Springer-Verlag Berlin Heidelberg 2017

\begin{abstract}
The impact of the self-attraction and loading effect (SAL) in a regional 2D barotropic tidal model has been assessed, a term with acknowledged and well-understood importance for global models but omitted for boundary-forced, regional models, for which the implementation of SAL is nontrivial due to its non-local nature. In order to understand the impact of the lack of SAL effects in a regional scale, we have forced a regional model of the Northwest European Continental Shelf and the North Sea (continental shelf model $(\mathrm{CSM})$ ) with the SAL potential field derived from a global model (GTSM), in the form of a pressure field. Impacts have been studied in an uncalibrated setup and with only tidal forcing activated, in order to isolate effects. Additionally, the usually adopted simple SAL parameterization, in which the SAL contribution to the total tide is parameterized as a percentage of the barotropic pressure gradient (typically chosen 10\%), is also implemented and compared to the results obtained with a full SAL computation. A significant impact on M2 representation is observed in the English Channel, Irish Sea and the west (UK East coast) and south (Belgian and Dutch Coast) of the North Sea, with an impact of up to $20 \mathrm{~cm}$ in vector difference terms. The impact of SAL translates into a consistent M2 amplitude and propagation speeds reduction throughout the domain. Results using the beta approximation, with an
\end{abstract}

This article is part of the Topical Collection on the 18th Joint Numerical Sea Modelling Group Conference, Oslo, Norway, 10-12 May 2016

Responsible Editor: Ulf Gräwe

Maialen Irazoqui Apecechea

Maialen.IrazoquiApecechea@deltares.nl

1 Deltares, P.O. Box 177, 2600 MH Delft, the Netherlands

2 Technical University of Delft (TU Delft), Delft, Netherlands optimal domain-wide constant value of $1.5 \%$, show a somewhat comparable impact in phase but opposite direction of the impact in amplitude, increasing amplitudes everywhere. In relative terms, both implementations lead to a reduction of the tidal representation error in comparison with the reference run without SAL, with the full SAL approach showing further impacted, improved results. Although the overprediction of tidal amplitudes and propagation speeds in the reference run might have additional sources like the lack of additional dissipative processes and non-considered bottom friction settings, results show an overall significant impact, most remarkable in tidal phases. After showing evidence of the SAL impact in regional models, the question of how to include this physical process in them in an efficient way arises, since SAL is a non-local effect and depends on the instantaneous water levels over the whole ocean, which is non-trivial to implement.

Keywords Northwest European Shelf · Self-attraction and loading $\cdot$ Tide surge

\section{Introduction}

The self-attraction and loading effect is a well-understood phenomena and has a widely acknowledged effect on the tidal dynamics in a global scale. This phenomenon consists of three effects: the deformation of the ocean seafloor due to the weight of the water column, the associated mass redistribution and corresponding changes in the gravitational field and the gravitational attraction of the water body on itself. The importance of this effect for global hydrodynamic models has been extensively discussed in several studies (Tamisiea et al. 2010) and is reported to be in the order of $10 \%$ of the barotropic pressure gradient, reducing errors in the sea surface modelling significantly (Stepanov and Hughes 2004). In particular, 
self-attraction and loading effect (SAL) is known to impact significantly the tidal phases (Gordeev et al. 1977) and therefore, the need to include this term in global tidal models is widely acknowledged. Extensive literature is available for the impact of SAL on the full spectrum of ocean variability, from rapid timescales (e.g. tides and atmospherically driven motions) to monthly and interannual time scales (Vinogradova et al. 2011).

The calculation of the SAL term is not trivial; it entails a very time-consuming convolution that can make its full online computation too expensive relative to the solving time of the ocean model equations (Stepanov and Hughes 2004). Hence, the choice of many authors for a simple proportionality constant (hereinafter denoted as the beta approximation, $\beta$ ) between SAL elevation and surface elevation, proposed by Accad and Pekeris (1978), with typical values of $\beta \sim 0.1$. Later on, Ray (1998) showed that this proportionality constant should be different for deep water than for shallow water and therefore, this approximation introduces large errors. Several authors afterwards have applied a fitted or depth dependent space-varying proportionality constant fields in the SAL parameterization (Shriver et al. (2012) employ a simplified scalar approximation of the SAL). However, Stepanov and Hughes (2004) and Kuhlmann et al. (2011) showed that this parameterization still generates significant errors due to the time-dependent nature of the sea surface anomaly associated to SAL, especially when atmospheric forcing is also considered, for which correlation seems to decrease dramatically.

Another approach used by Le Provost et al. (1998) and Egbert and Erofeeva (2002) consists on estimating the SAL periodic tide from modern estimates of elevations and introduces it as such in the momentum equations. However, this approach leads in general to inconsistencies between the SAL and the computed elevation, triggering significant imbalances in the energy calculations. Additionally, this approach fails to incorporate possible changes in ocean geometries which would entail different tidal elevations to the available modern estimates. Hendershott (1972) proposed an iterative method used by several studies like Jayne and St Laurent (2001), Arbic et al. (2004) and Egbert et al. (2004). This iteration is possible in tide-only runs in which the SAL is periodic, and therefore can be expressed in terms of meaningful amplitudes and phases, but fails when incorporating SAL effects of non-periodic motions (Ngodock et al. 2016). These last two approaches, although they incorporate SAL effects at each time step, fail on a full SAL representation due to the lack of spatial integration from instantaneous water levels.

SAL has received a lot of interest in the last decade not only for its effect on global sea surface dynamics but also for its effect in bottom pressure and therefore the interpretation of bottom pressure measure by, e.g. satellite geodesy missions like Gravity Recovery and Climate Experiment (GRACE).The response of the ocean to the SAL forcing is in equilibrium for long periods, and can therefore be computed offline, but for short periods, the non-equilibrium part of the response is expected to be significant and therefore very meaningful for interpreting measurements from GRACE for which it is necessary to model mass redistributions occurring at periods less than about 2 months. The physics of SAL has also been of interest for several decades particularly because of the associated spatially varying long-term trends in sea level that can result from the melting of land ice. An accurate representation of SAL effects corresponding to variable melting rates of glaciers and ice sheets at interannual scales, even if typically smaller, serves not only for a better understanding of sea level dynamics and measurements from GRACE but also as important indicators of climate change (Vinogradova et al. 2011).

While the importance of the inclusion of this phenomenon in global scale tidal models is strongly acknowledged, it is generally omitted in regional models, including shelf-wide models. Since most of these models use boundary conditions obtained from global tidal models which do include this effect, the contribution of the SAL is limited to the boundary forcing and not implemented as a body force internally in the domain. While this simplification is valid for coastal models in which the tidal solution is mainly determined by the boundary conditions, in a similar manner to the tide generating potential, the SAL potential plays an increasingly important role on the propagation of the tides as the size of the model increases, extending towards the deeper ocean. Only very recent literature has been found to address the possible implications of the omission of SAL effects at a regional scale (Teng et al. 2016). Teng et al. (2016) show the importance of the inclusion of SAL effects in the numerical modelling of semi-diurnal tides in the seas around Japan and East China, defining the term as a sum of the harmonic motions representing the periodic SAL anomaly associated to each tidal constituent. The harmonic constants of the SAL tides are taken from a preceding study in the same area by Fang et al. (2013). Although the study of Teng et al. (2016) is limited to tide, and only the periodic semidiurnal SAL tides are included in the model, it shows how significant these effects can be (up to $30 \%$ of the tidal signal in certain locations) and how spatially variable this contribution relative to the astronomical tide is. Errors associated to neglecting the SAL contribution are thought to be commonly tuned off by calibration of, e.g. bathymetry and bottom roughness. However, we believe that including more physical processes in the model and thereby reducing the need for calibration is inherently better, as this increases system understanding and enhances the validity of the model for a wider range of applications or scenarios.

In this paper, we address the question of how important the SAL effect is in a regional model of the Northwest European Shelf (and the North Sea in particular) and analyse the sensitivity of the tidal solution to the inclusion of this phenomena. Since the SAL solution is non-local but requires a spatial integration over the whole ocean, this is performed by computing the SAL potential field in time from a global model to then apply it as a surface forcing to the regional model. 
In the following sections, a description of the two models used in this experiment is presented, followed by an explanation of the methodology for the SAL calculation and subsequent forcing in the regional model. A thorough analysis of the results is performed by looking at the impact of SAL in different areas of the regional model, identifying trends and drawing conclusions on the importance of SAL inclusion as a body force internally in shelf-wide regional tide models.

\section{Model description and SAL parameterization}

In this section, the model used for the SAL field calculation is described, alongside the analytical solution for the selfattraction and loading phenomena and methodology followed for its numerical implementation in the model.

\subsection{The global tide surge model}

The global tide surge model (GTSM) is a global, unstructured barotropic model for tide surge based on the Delft3D FM software developed by Deltares (Kernkamp et al. 2011). DFlow FM is a multi-dimensional (1D, 2D and 3D) hydrodynamic (and transport) modelling software which calculates non-steady flow and transport phenomena that result from tidal and meteorological forcing on structured and unstructured, boundary fitted grids, both in Cartesian and in spherical coordinates. The term Flexible Mesh in the name refers to the flexible combination of rectilinear or curvilinear grids and unstructured grids composed of triangles and/or quads (Delft3D FM User's Manual 2016). It is precisely the unstructured capability that makes it attractive for domains covering from deep waters to coastal, shallow waters, in order to have a varying grid size and perform efficient large-scale computations, such as for a global model.

When going from regional to global scale, several terms need to be included in the hydrodynamic equations which are non-negligible any more. These terms are described below and included in the momentum equation (Eq. (5)). Unlike regional tidal models which are forced through tidal open boundary conditions, in a global model, the tidal movement is purely induced by the tide generating forces. These can be expressed in terms of equilibrium $\operatorname{tide}\left(\xi_{\mathrm{EQ}}\right)$ as follows:

$\xi_{\mathrm{EQ}}=\frac{\Phi\left(1+k_{2}-h_{2}\right)}{g}$

Where $g$ is the gravity and $\Phi$ is the potential due to the gravitational pull of the celestial forces. The terms $k_{2}$ and $h_{2}$ are the Love numbers associated to the Earth's surface displacement due to these same forces, commonly known as solid Earth tides. These terms are implemented in the computational core following
Schrama (2007). This forcing is applied in a $1^{\circ}$ resolution grid and interpolated to the computational grid at each time step.

Another physical process typically omitted in regional models but of noteworthy importance in a global scale is the dissipation of barotropic energy through generation of internal tides when tides propagate over steep topography in stratified, deep waters $\left(\tau_{\text {IT }}\right)$. While dissipation through bottom friction remains the main dissipation mechanism in shallow waters, the dissipation through internal tides drag is the dominant mechanism for deep waters. It accounts for one fourth of the global barotropic energy dissipation $(\sim 1 \mathrm{TW})$, and significantly affects the propagation of tides and energy distribution around the globe. In the GTSM, an anisotropic numerical implementation is included in order to allow for dissipation only in the direction of the steep bathymetry and not along isobaths. The formulation used is according to Maraldi et al. (2011), and is a function of the buoyancy frequency (Brunt Väisälä frequency), local velocity, local bathymetry gradient and a user-defined coefficient:

$\boldsymbol{\tau}_{\mathrm{IT}}=-C_{\mathrm{IT}} N(\nabla h \times \mathbf{u}) \nabla h$

where $C_{\mathrm{IT}}$ is the user-defined coefficient, $N$ is the depthaveraged Brunt Väisälä frequency, $\nabla h$ is the local bathymetry gradient and $\mathbf{u}$ is the local velocity vector. Since the GTSM is a barotropic, depth-averaged model and the buoyancy frequency is dependent of the local stratification, the depth-averaged Brunt Väisälä frequency is pre-computed from monthly means of temperature and salinity fields and implemented as a spatially varying scalar field, constant in time. This means that seasonal patterns in dissipation through internal tides generation are not represented. However, for this study, the simulation period is of 2 months (January and February) and stratification temporal variability is considered negligible. The temperature and salinity fields are therefore calculated as a mean between January and February monthly means.

For the dissipation through bottom friction ( $\tau$ ), a Chézy quadratic formulation is used with a typical constant bottom friction coefficient of $C_{\mathrm{D}}=62.65$ over the whole domain, as follows:

$\tau={ }^{g} / C_{\mathrm{D}}{ }^{2}\|\mathbf{u}\| \mathbf{u}$

All the terms explained previously are introduced appropriately in the momentum equation, leading to the following governing equations:

$$
\begin{aligned}
\frac{\partial \mathrm{h}}{\partial \mathrm{t}}+\nabla \cdot(h \mathbf{u})=0 \\
\frac{\partial \mathbf{u}}{\partial \mathrm{t}}+\mathbf{f} \times \mathbf{u}+\frac{1}{\mathrm{~h}}(\nabla \cdot(h \mathbf{u u})-\mathbf{u} \nabla \cdot(h \mathbf{u})) \\
=-g \nabla\left(\xi-\xi_{\mathrm{EQ}}-\xi_{\mathrm{SAL}}\right)+\nabla \cdot\left(\nu\left(\nabla \mathbf{u}+\nabla \mathbf{u}^{\mathrm{T}}\right)\right)+\frac{\boldsymbol{\tau}}{h} \\
\quad+\frac{\boldsymbol{\tau}_{\mathrm{IT}}}{h}
\end{aligned}
$$


where $\mathbf{f}$ is the Coriolis force, $h$ is the water depth, $\nu$ is the (horizontal) viscosity and $\xi$ is the water level. The term $\xi_{\mathrm{SAL}}$ refers to the SAL effect which is explained in Sect. 2.2.

The GTSM has a spherical grid with cell size dependent on the (square root of the) bathymetry (courant grid refinement). In deep waters, the resolution is $\sim 50 \mathrm{~km}$ and it increases to up to $\sim 5 \mathrm{~km}$ at the coast. Additionally, a bathymetry gradientbased refinement is applied in order to properly represent topographic features in deep water-like ridges and trenches and allow for realistic local barotropic dissipation through internal tides generation. With this additional refinement, the total number of nodes is 1.7 million. Bathymetry used in the model is the GEBCO 2014 dataset, defined in a 30" regular grid (http://www.gebco.net/data_and_products/gridded bathymetry_data/) in both sea and land. The computational time step used in GTSM is $150 \mathrm{~s}$.

The model's time-stepped nature and hence capability to simulate total water levels resulting from both tidal and meteorological forcing (amongst others) makes it attractive for applications of, for example, storm surge (operational) forecasting, satellite data reduction for instantaneous water levels and vertical referencing. GTSM is already being used for the development of a global operational forecasting system (the Global Storm Surge Forecasting and Information System (GLOSSIS), Verlaan et al. 2015) and has been used in a historical global reanalysis of storm surges (Muis et al. 2016).

\subsection{Implementation of self-attraction and loading}

Self-attraction and loading effects induce a vertical displacement of the geoid relative to the seafloor. The displacement induced by a unit point load at an angular distance $\alpha$ from the reference position that can be defined analytically through the following Green function (Farrell 1973):

$G(\alpha)=\left(a / m_{\mathrm{E}}\right) \sum_{n=0}^{\infty}\left(1+k_{\mathrm{n}}^{\prime}-h_{\mathrm{n}}^{\prime}\right) P_{\mathrm{n}}(\cos \alpha)$

where $\alpha$ is the angular distance from the point load, $a$ is the Earth's radius and $m_{\mathrm{E}}$ is the mass of the Earth. $P_{\mathrm{n}}$ is the Legendre polynomials or order $\mathrm{n}$ used to express the inverse distance, and $k_{\mathrm{n}}^{\prime}$ and $h_{\mathrm{n}}^{\prime}$ are the elastic loading Love numbers (for gravitational changes in response to the load and deformation of the seafloor under the additional mass, respectively) that are defined according to the chosen Earth model. In this case, the Preliminary Reference Earth Model (PREM) by Dziewonski and Anderson (1981) is used. The PREM model is widely used for the investigation of loading due to ocean tide (Wang et al. 2012). The net effect of SAL is represented by the factor $\left(1+k_{\mathrm{n}}^{\prime}-h_{\mathrm{n}}^{\prime}\right)$.

Since computing the 2D convolution on the whole sphere (globe) is numerically costly, the Green function in Eq. (6) is implemented in GTSM using spherical harmonic functions, which allow to calculate the convolution on the sphere as a simple multiplication in the frequency domain. Additionally, separation of variables is used for the calculation of the spherical harmonic coefficients. These approaches allow for a more efficient calculation of the SAL term and a computationally affordable online calculation at each time step in GTSM.

The SAL potential $\Phi_{\mathrm{SAL}}$ is included in the model analogously to the tidal potential generated by the celestial forces, i.e. calculating the associated "SAL tide" $\xi_{\mathrm{SAL}}=\frac{\Phi_{\mathrm{SAL}}}{g}$ and including this term in the momentum equation (see Eq. (5)).

The method consists on using the water levels modelled at a time $n\left(\xi^{n}\right)$ to calculate the SAL tide $\xi_{\mathrm{SAL}}^{n}$ using the spherical harmonics approach, and introduce this term in Eq. (5) to calculate the new $\xi^{n+1}$ in the following time step. Unlike the iterative solution approach proposed by Hendershott (1972), this methodology avoids any convergence problems or artificial work by the SAL term due to inconsistencies between the SAL solution and the instantaneous water levels.

The SAL term is calculated at each time step in the same $1^{\circ}$ regular grid as for the tide generating term in the GTSM and fed back into the model in the following time step. As a way of verification for the SAL, used in several other global tidal studies (Hendershott 1972; Egbert et al. 2004), the total work done by the SAL term averaged over a tidal cycle showed to be zero.

As a reference, the so-called beta approximation $(\beta)$ mentioned earlier would be implemented as a reduction of the barotropic pressure gradient:

$\nabla \xi_{\mathrm{SAL}}=\beta \nabla \xi$ with $\beta=0.1$

which leads to a linear relationship between the water levels and the so-called SAL tide term:

$\xi_{\mathrm{SAL}}=\beta \xi+$ const

Although there is currently no functionality for using a strict beta approximation in GTSM, a simple way to simulate its effect is to reduce gravity by a factor $(1-\beta)$ :

$$
\begin{aligned}
\frac{\partial \mathbf{u}}{\partial \mathrm{t}}+ & \mathbf{f} \times \mathbf{u}+\frac{1}{h}(\nabla \cdot(h \mathbf{u u})-\mathbf{u} \nabla \cdot(h \mathbf{u})) \\
= & -g(1-\beta) \nabla\left(\xi-\xi_{\mathrm{EQ}}\right)+\nabla \cdot\left(\nu\left(\nabla \mathbf{u}+\nabla \mathbf{u}^{\mathrm{T}}\right)\right)+\frac{\boldsymbol{\tau}}{h} \\
& +\frac{\boldsymbol{\tau}_{\mathrm{IT}}}{h}
\end{aligned}
$$

This simplified formulation is used in this experiment in order to study the impact of using a full SAL calculation in comparison to simple parameterizations.

Parallelization has been implemented in the routine for calculating the spherical harmonic coefficients, which significantly helped on speeding up the computation. This 
implementation has proven to impact minimally the computational times, and therefore, its online computation has been implemented permanently in the model.

\subsection{Regional model: the continental shelf model}

Due to the threat the storm surges represent for the North Sea, all the countries surrounding it have their own national storm surge forecasting service, based on numerical models. The Netherlands uses the so-called Dutch Continental Shelf Model, which has undergone throughout years an exhaustive development and rigorous optimization, leading to the DCSM version 6. The Dutch Continental Shelf Model (v6) is a realtime storm surge forecasting 2D model for the Northwest European Continental Shelf and North Sea run operationally by the Dutch Ministry of Water and Defence (Rijkswaterstaat) in the Netherlands. A detailed explanation of the model development and especially the rigorous reduction in uncertainty in the time-independent parameters can be found from Zijl et al. (2013). Reduction in uncertainty in the meteorological forcing was later achieved through the integration of operationally available observational data by means of a steady-state Kalman filter (Zijl et al. 2015), and nowadays, it is considered one of the most accurate shelf-wide storm surge models available. In the present paper, the deterministic version of this model is taken as a reference for the regional model used.

This study aims to look at effects of SAL in the North Sea and potentially impacted surrounding seas in the Northwest European Shelf. Since the SAL effects are likely compensated for by the calibration of the model, we use a shelf-wide model based on an uncalibrated version of the DCSMv6, which we will refer to hereinafter as continental shelf model (CSM). We use as bathymetry the recently available EMODnet dataset (http://www.emodnet-hydrography.eu). An uncalibrated, constant roughness value is used for the whole domain in order to make an assessment of the impact of the SAL term before any possible calibrations. The bottom friction parameterization is based on a Manning quadratic formulation, with constant Manning coefficient of 0.028, which is of the order of values used for similar applications.

The grid of CSM covers the area between $43 \mathrm{~N}$ and $63 \mathrm{~N}$ in latitude, and $15 \mathrm{~W}$ and $13 \mathrm{E}$ in longitude .The grid is in spherical coordinates, with a uniform grid resolution of $1 / 40^{\circ}$ in the longitude direction and $1 / 60^{\circ}$ in the latitude direction, resulting in a grid cell of approximately $1 \mathrm{~nm}$ in both directions. The model is forced at the northern, western and southern boundaries by specifying 38 tidal constituents in the frequency domain obtained from FES2012. The tidal signal is then derived through a harmonic expansion at each of the 205 boundary support points and linearly interpolated to intermediate grid boundary points. The global tide model FES2012 already includes the SAL term (Lyard et al. 2006), and therefore, the SAL effect is already included at the boundary forcing in the CSM. The computational time step used is $120 \mathrm{~s}$.

In this preliminary study of effects of SAL in regional models, only tides are going to be modelled for simplicity and because it is eventually the tides that are expected to be mostly affected by this term. Impacts on surge are out of the scope for this preliminary sensitivity study, as are other loads like hydrology. Therefore, only tidal forcing is used in this experiment (besides SAL forcing) and meteorological forcing is not considered.

\section{Methodology}

The methodology for this experiment is the following: first, the GTSM is run for 2 months and SAL potential fields are outputted every $30 \mathrm{~min}$. Second, the SAL field is transformed into a pressure field and interpolated to a regular grid. Thirdly, the SAL pressure field is applied as atmospheric pressure forcing in the CSM model and a tidal run of the corresponding period is performed. Finally, results are analysed through harmonic analysis.

The period chosen for the simulations is from January 2007 to February 2007. Since in this study the objective is to look at short-term, dynamic effects of the SAL on the tides, a short period is sufficient. However, since a harmonic analysis is wanted over the simulated water levels to assess results in the frequency domain, a minimum of 2 months of time series is recommended in order to be able to capture lower frequency constituents and perform a better separation of the diurnal and semi-diurnal constituents.

Furthermore, a test case with the (simplified) beta approximation as given in Eq. (9) is also performed, in order to compare the direction of the impact when using this parameterization in comparison to solving the spherical harmonics at each time step.

\subsection{SAL field from GTSM}

The SAL (potential) field is outputted on the global model at a temporal resolution of $30 \mathrm{~min}$. This is deemed sufficient to capture the relevant periodicity in the SAL forcing.

In order to demonstrate that the linear beta approximation is insufficient to represent the spatial and temporal characteristics of the SAL field, the linear statistical relationship between the SAL field and the water level field is analysed (see Eq. (8)). For this, we calculate two quantities: the optimal linear $\beta$ coefficient and the correlation between the two variables. The former will provide a picture of the spatial variability and scale of a fitted $\beta$ linear coefficient, and the latter will present the degree of linear dependency between the two variables. The two quantities are calculated at each grid cell in a 
period of 2 months, making use of the map output mentioned previously.

The optimal $\beta$ coefficient map is calculated using a linear regression, with $\xi_{\mathrm{SAL}}$ as the dependent variable and $\xi$ as the explanatory variable, through the following equation:

$\beta=\frac{\operatorname{cov}\left(\xi_{\mathrm{SAL}}, \xi\right)}{\operatorname{var}(\xi)}$

where $\operatorname{cov}()$ is the covariance and $\operatorname{var}()$ is the variance.

Figure 1 shows the optimal beta coefficient in the whole globe, alongside a detail of the field in the Northwest European Continental Shelf. One can see that the beta field is somewhat uniform in deep waters, but clearly non-uniform on the shelves and coastal waters. In general, higher values of beta of around $0.1-0.15$ are needed in deep waters, and lower values at the coast. In some places, this coefficient reaches even negative values. For the North Sea, the non-uniformity is evident and the range of values for beta is wide, showing that there is no visible correlation between depth and beta values.

Once the spatial variability of the optimal beta is characterized, we have a look at the temporal variability. Correlation is computed as the division of the covariance of the two variables (SAL tide, water levels) by the product of their standard deviation for each computational cell:

Corr $=\frac{\operatorname{cov}\left(\xi_{\mathrm{SAL}}, \xi\right)}{\sigma_{\xi} \cdot \sigma_{\xi_{\mathrm{SAL}}}}$

where $\sigma_{\xi}$ is the standard deviation of the water level and $\sigma_{\xi_{\mathrm{SAL}}}$ is the standard deviation of the SAL tide. Figure 2 shows the correlation between the two variables in the whole globe, with a detail of the North Sea again. Correlation is very high in deep waters, but proves to be poor in continental shelves and semi-

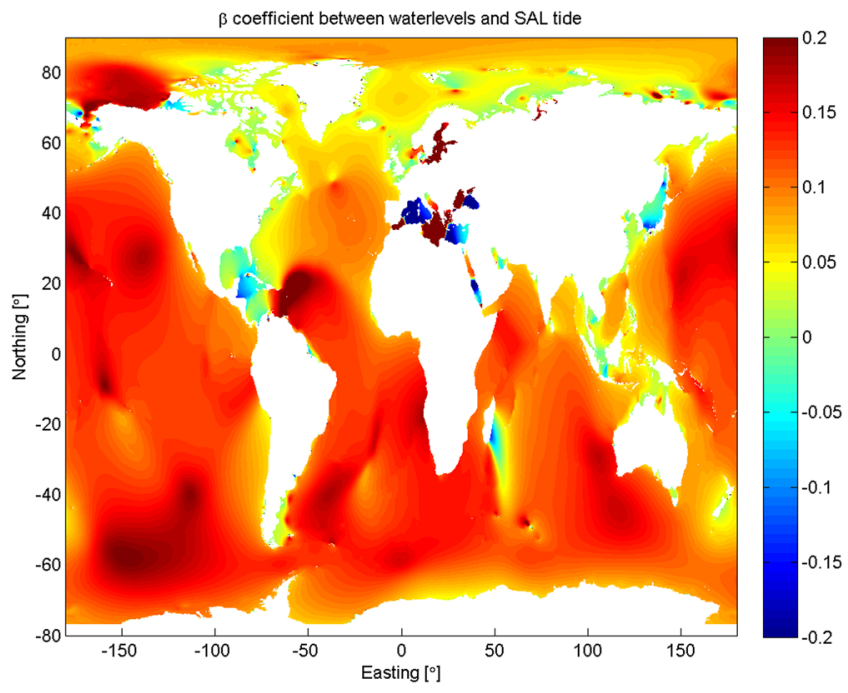

Fig. 1 Computed $\beta$ field from GTSM-modelled water levels and SAL tide enclosed seas. In places where the tidal amplitude is low (e.g. Mediterranean Sea, Caribbean Sea), correlation is also very poor. For the North Sea, the low correlation shows that even a spatially varying, optimal beta field fails in the representation of the SAL effect for several regions.

These results show the need of introducing the SAL effect as non-local and non-uniform, if one wants to account for SAL effects in shallow waters.

\subsection{Implementation of SAL field in a regional model}

As mentioned previously, the SAL effect is introduced in the regional CSM model for the experiments in this paper through a conversion of the field from potential to pressure $\left(p_{\mathrm{SAL}}\right)$, using the following equation:

$p_{\mathrm{SAL}}=\rho g \xi_{\mathrm{SAL}}=g \Phi_{\mathrm{SAL}}$

where $\Phi_{\mathrm{SAL}}$ is the SAL potential field. This field, outputted at the cell centres of the unstructured GTSM computational grid, is interpolated to a regular grid with a resolution of $11 \mathrm{~km}$ approximately and used to force the regional model.

\subsection{CSM runs with SAL forcing}

For the assessment of the impacts of SAL forcing on the tides, the results are analysed both through harmonic analysis at observation stations and through co-tidal charts, in order to get an idea of the spatial changes in tidal propagation and modulation.

At the tide gauge locations, modelled tidal constituents are compared against those derived from measurements. The location of the tide gauge stations and the source of the measurements are described in detail by

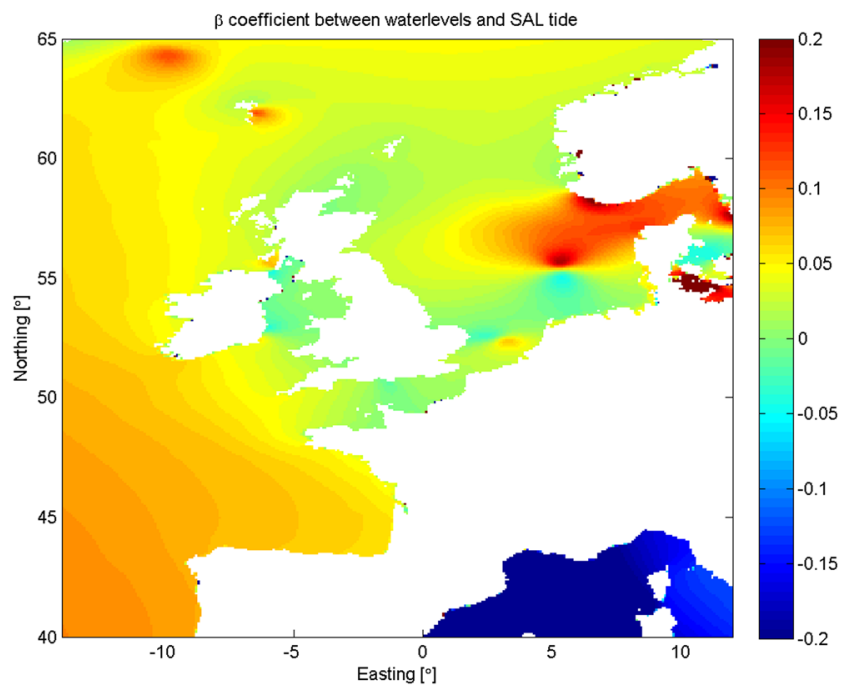



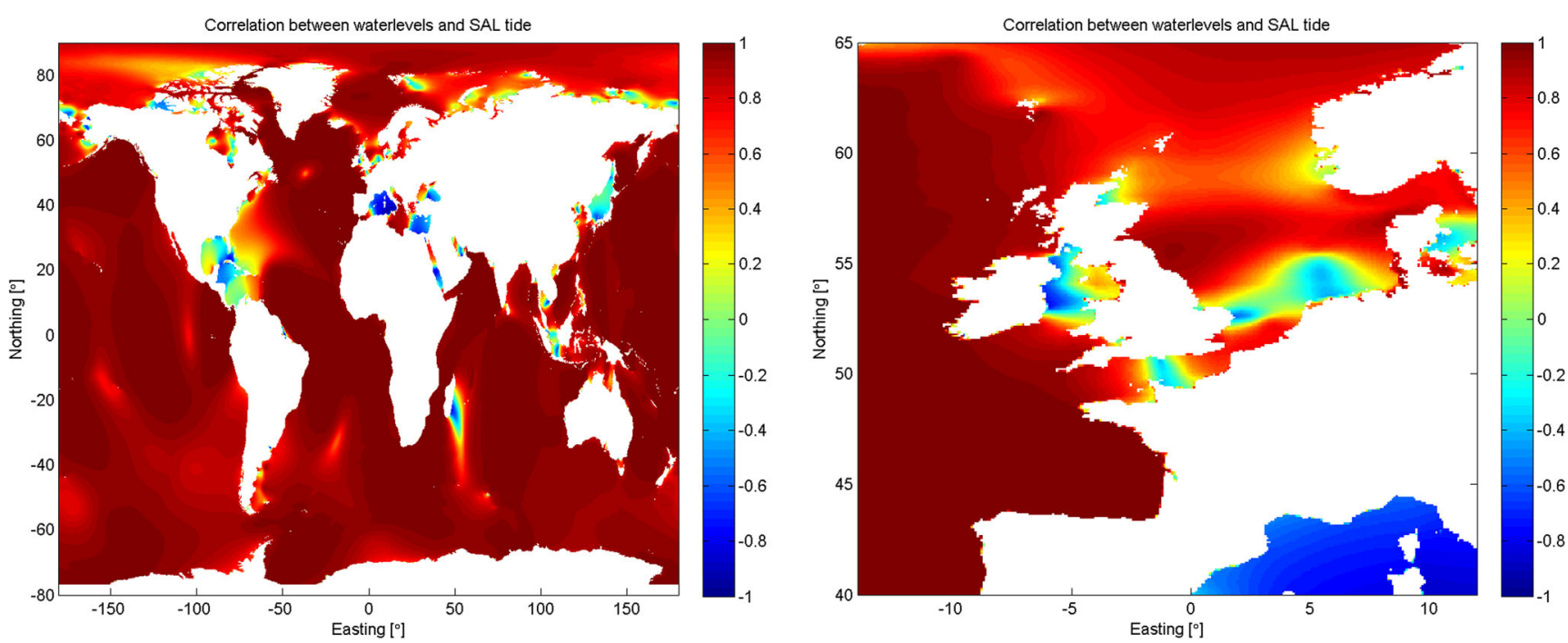

Fig. 2 Computed correlation between GTSM-modelled water levels and SAL tide

Zijl et al. (2013). Since the source model in which the CSM is based is the operational model for the Netherlands, special attention is going to be paid to the effect of the SAL term in the Dutch coastal stations, depicted in Fig. 3.

For harmonic analysis, the program T_TIDE (Pawlowicz et al. 2002) was used, with a set of 23 tidal constituents (for series covering a 2-month period). Both amplitudes and phase errors and sensitivity will be assessed, and combined into a vector difference (Le Provost et al. 1995) to evaluate total errors in the frequency domain.

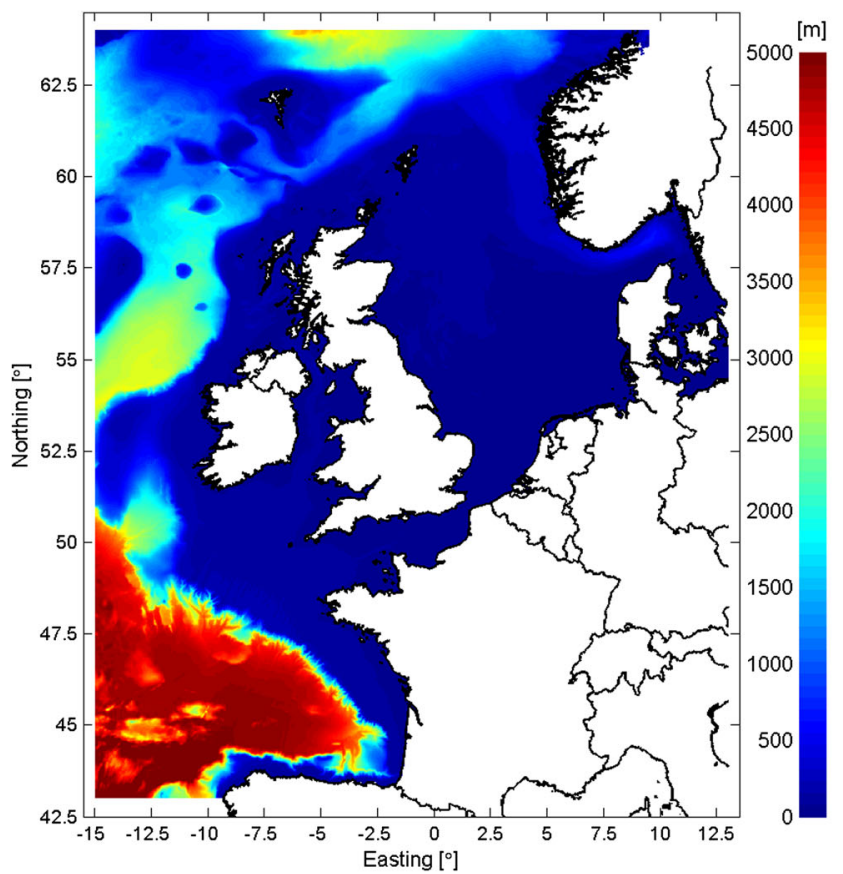

\section{Results}

\subsection{Vector difference charts}

Firstly, in order to get an idea of how the inclusion of SAL affects the tidal representation spatially and where in the domain it is more interesting to have a thorough look, we compare modelled amplitudes and phases spatially for separate tidal constituents. Changes due to SAL inclusion are expected to be non-negligible, but not of an order of magnitude for which impacts of

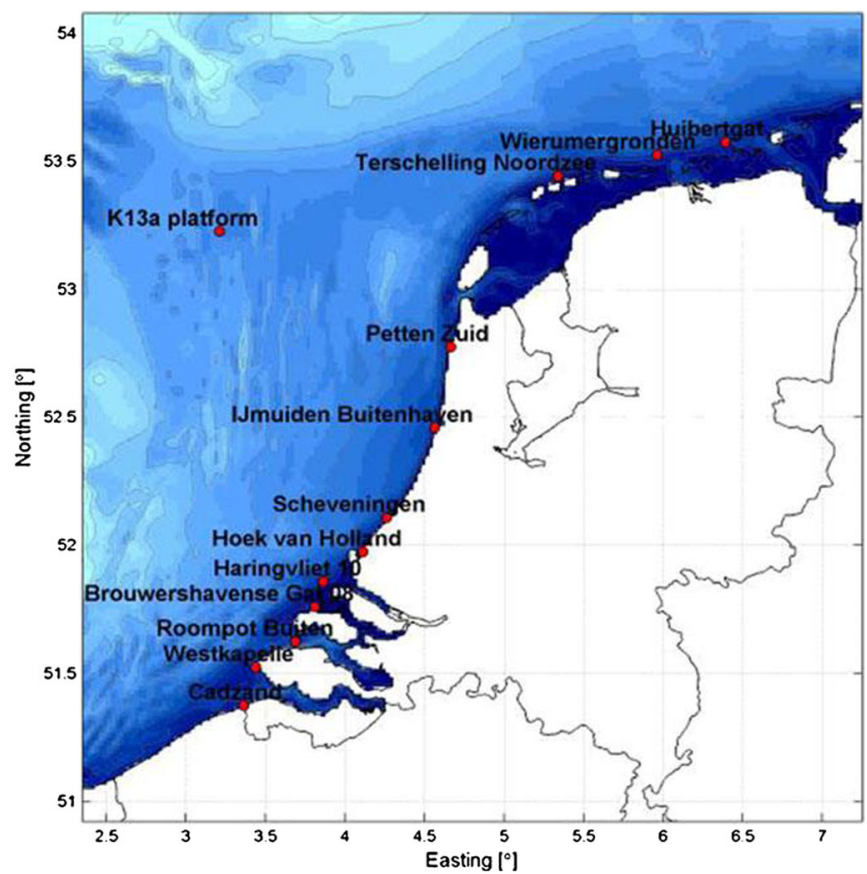

Fig. 3 CSM model extent and bathymetry (left). Thirteen representative tide gauge stations for the Dutch Coast (right) 
SAL inclusion in co-tidal charts will be evident to the reader's eye. Furthermore, the slight displacement of amphidromic points makes the interpretation of the individual amplitude $(H)$ and phase $(G)$ difference maps difficult. Therefore, we focus on presenting the impact by computing the vector difference (VD) at each grid cell between the simulation with $\operatorname{SAL}\left(H_{\mathrm{SAL}}, G_{\mathrm{SAL}}\right)$ and
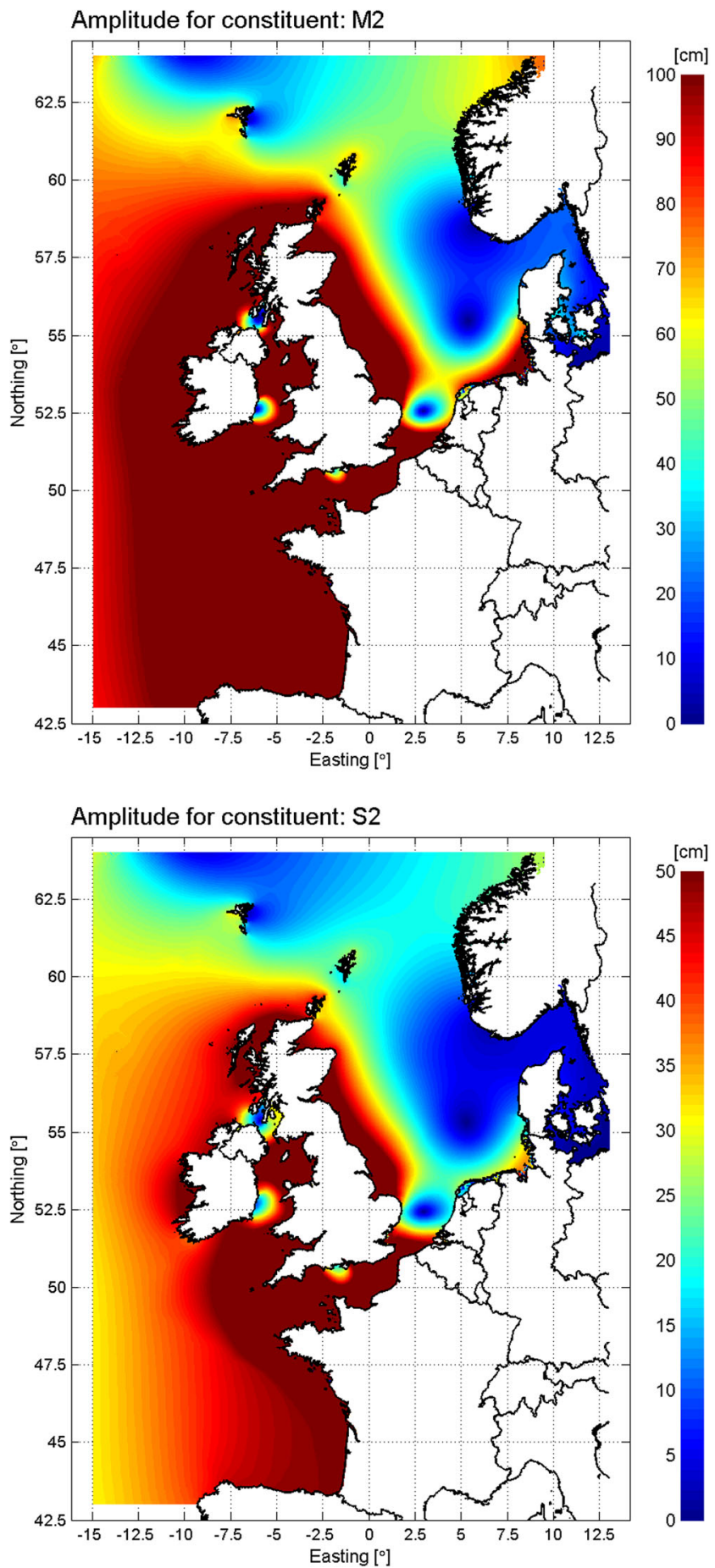

the simulation without $\operatorname{SAL}(H, G)$, and creating a vector difference map.

$\mathrm{VD}=\sqrt{\left[H_{\mathrm{SAL}} \cos G_{\mathrm{SAL}}-H \cos G\right]^{2}+\left[H_{\mathrm{SAL}} \sin G_{\mathrm{SAL}}-H \sin G\right]^{2}}$

Figure 4 shows the vector difference spatially between the two cases for the M2 and S2 constituents, chosen for showing
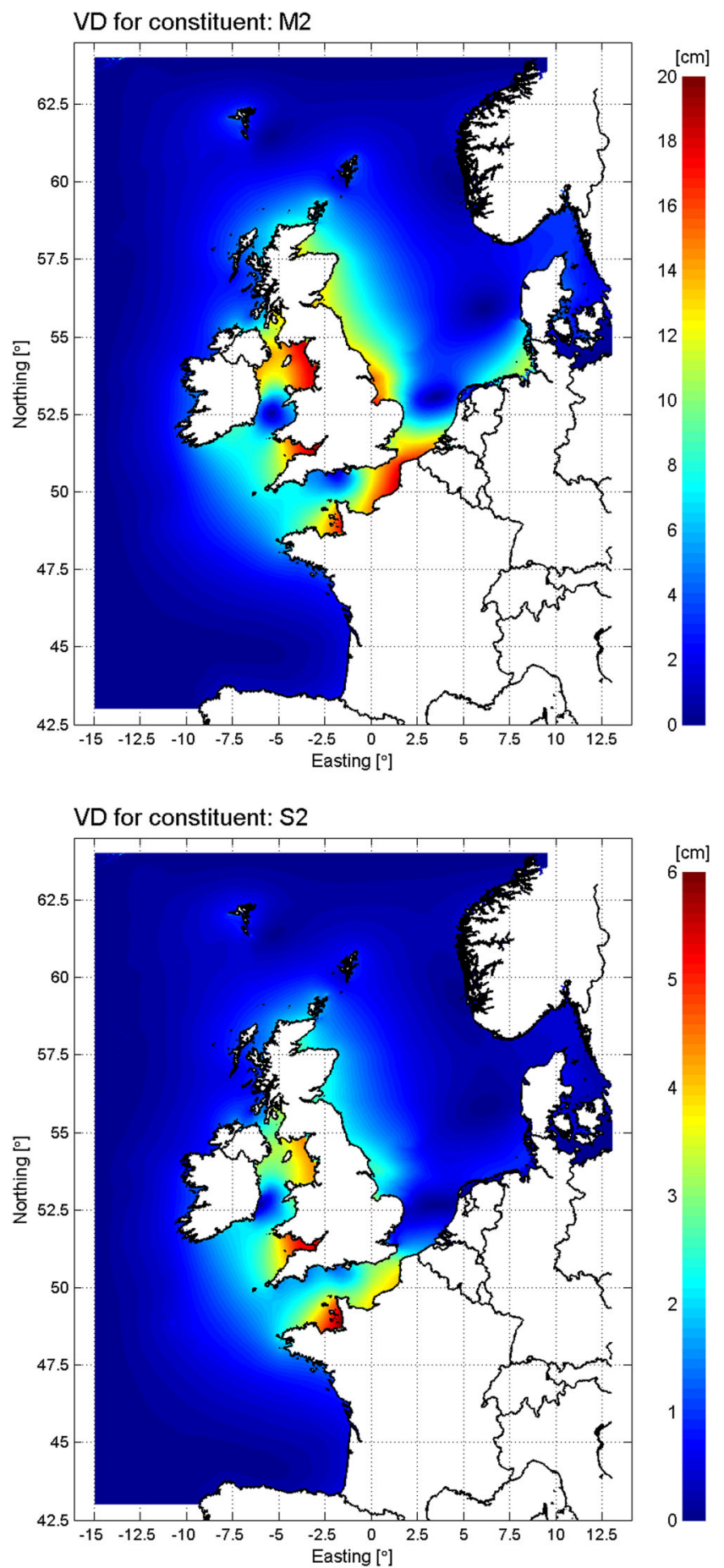

Fig. 4 Left: amplitude charts for M2 (top) and S2 (bottom) in centimetres. Right: corresponding vector difference plots (cm) from amplitude and phase charts for cases with and without SAL 
the biggest impact. M2 shows to be the most impacted constituent, expected for being the main constituent in these waters. The vector difference change for this constituent reaches values of $20 \mathrm{~cm}$ in the English Channel and in the Irish Sea, and high values as well at the Western and Southern North Sea.

For S2, the second most impacted constituent, impact reaches values of $6 \mathrm{~cm}$ at the southern entrance of the English Channel and in the Celtic Sea (especially in the Bristol Chanel). Relatively high values are also shown in the east coast of the Irish Sea $(\sim 4 \mathrm{~cm})$.

For the rest of constituents (N2, M4 maps), the scale of the impact is much smaller, with maximum values reaching $4 \mathrm{~cm}$ very locally.

For illustration of the impact, we show the time series for a period of a week in one of the stations significantly affected by the SAL addition, Cadzand, in the coast of the Netherlands. Figure 5 shows the modelled tide time series against the observed tidal signal for the cases without SAL and with SAL. The residual is also plotted for a clearer visualization of the changes from one to another. From this plot, the positive impact in phase due to SAL is evident, as well as the reduction of the previously overestimated amplitudes.

Based on the impact co-tidal charts, we will look in more detail at the SAL impact on the M2 representation in the English Channel, Irish-Celtic Sea and North Sea, making a comparison at the observation stations in the different areas against tide gauge data-derived M2 component. A more detailed look is taken at the Dutch Coast, of evident relevance for this particular model. Location and names of the observation stations, as well as extent of each considered area, are available in the Appendix A. The area considered for the Irish Sea extends further southwards, into the Celtic Sea.

The amplitude and phase errors $(\Delta H, \Delta G)$ presented in the following sections correspond to computed (c) values minus observed $(o)$ values $\left(H_{\mathrm{c}}-H_{\mathrm{o}}, G_{\mathrm{c}}-G_{\mathrm{o}}\right)$, respectively.

\subsection{Results for the observation stations}

When comparing computed against observed time series, the Root Mean Square (RMS) impact of SAL is of $1.2 \mathrm{~cm}$. The major contributor to this impact is the M2 constituents, and therefore we will focus on this constituent for the presentation of results hereinafter. The M2 vector difference reduces $1.6 \mathrm{~cm}$ (RMS). Although the impact might look small when averaged throughout all the stations in the time domain, the spatial distribution of this impact shows a very different picture. Figure 6 shows the amplitude and phase difference due to the addition of the SAL forcing, calculated as $\left(H_{\mathrm{SAL}}-H_{\mathrm{no} \mathrm{SAL}}\right)$ and $\left(G_{\mathrm{SAL}}-\right.$ $G_{\text {no SAL }}$, respectively. One can observe a trend of decreasing amplitudes and increasing phases due to SAL effect.

The following is a description of the results at the observation stations divided per area, namely the English Channel, Irish Sea (extended), North Sea and lastly, the Dutch Coast. A detailed breakdown of the M2 representation for each station and area, sorted out by direction of propagation of the M2 constituent, can be found in the tables in Appendix B. A total RMS error and bias is included for amplitude and phase in each table.

\subsubsection{Results for the English Channel}

In the time domain, the total RMS error when compared to observations reduces in $4.5 \mathrm{~cm}$, with an average reduction of error per station of $5.2 \mathrm{~cm}$. The harmonic analysis confirms what is depicted by Fig. 4: M2 is the main contributor to this impact, with its vector difference reducing in $7.6 \mathrm{~cm}$. S2 also improves significantly, with a reduction of the vector difference of $1.6 \mathrm{~cm}$. The impact of SAL translates into an improvement of the M2 representation in every station, with a remarkable impact and improvement in the stations of Le Havre, Dover and Boulogne Sur Mer. These stations are situated in the southern coastal stretch of the channel, consistent with the SAL impact shown in Fig. 4. Figure 7 shows a plot of the amplitude and phase impact for each station.

For the case without SAL, the results showed a consistent too fast propagation (negative phase errors) for all the stations, which is improved everywhere with the SAL inclusion by slowing it down. When it comes to amplitudes, the impact is also directionally consistent throughout the stations, with a reduction of amplitude everywhere due to SAL, although not necessarily leading to better amplitudes everywhere. For
Fig. 5 Observed tidal signal (black), modelled tidal signal with SAL (blue) and without (red) for a period of a week in Cadzand, in the coast of the Netherlands. Residuals are plotted in the corresponding colours

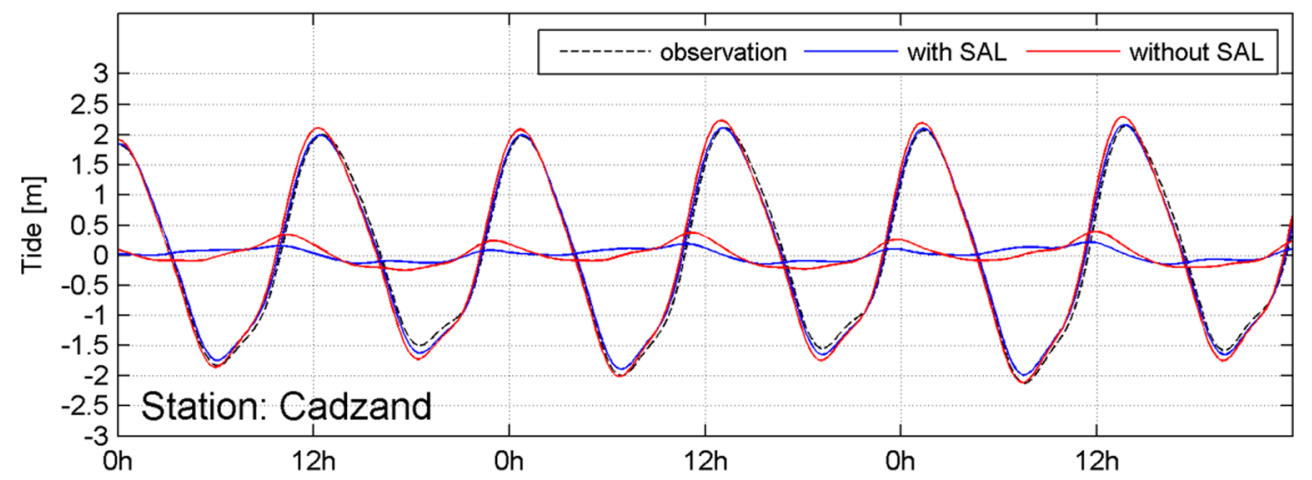



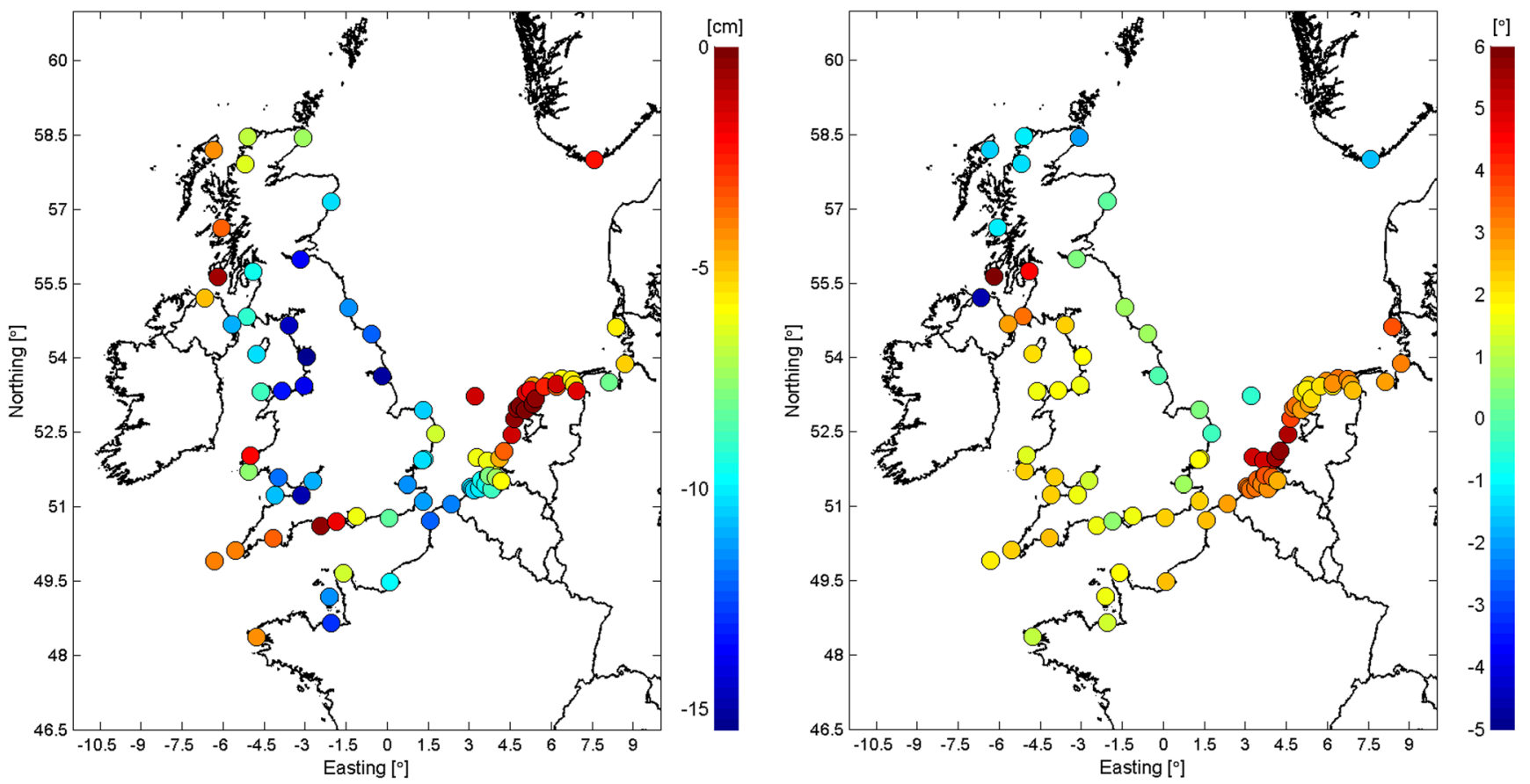

Fig. 6 Impact of SAL term in M2 amplitude $\left(H_{\mathrm{SAL}}-H_{\text {no SAL }}\right.$, left $)$ and phase $\left(G_{\mathrm{SAL}}-G_{\mathrm{no}} \mathrm{SAL}\right.$, right $)$ in all observation stations

the stations furthest south in the channel, amplitudes deteriorate due to underestimation once the SAL is added; north of Cherbourg onwards; however, amplitudes were considerably overestimated, and SAL alleviates this. See Table 5 in Appendix B for results per station.

\subsubsection{Results for the Irish Sea}

For the Irish Sea, although the overall RMSE does not vary much $(0.4 \mathrm{~cm})$, there is a big impact in the propagation of the M2 from station to station. Due to the existence of amphidromic points in this sea, the direction of the impact is not the same for all the stations: the direction of the impact in phase is different for the northern stations than for the southern ones, as shown in Fig. 8. Slight changes on the location of these amphidromic points will affect the results from harmonic analysis in the individual stations significantly, potentially in both amplitude and phase. An example of this is Port Ellen, located right nearby an amphidromic point, which experiences a drastic phase error change in magnitude and sign. Other stations around the same amphidromic point like Portrush and Bangor also show an important reduction of the phase error and also an important reduction of the amplitude error, pointing to a better representation of the amphidromic point.

The impact of the SAL translates in a reduction of amplitude in all the stations. In the original case, amplitudes were considerably underestimated in the southern part of the Irish Sea, up to Fishguard, considerably overestimated from
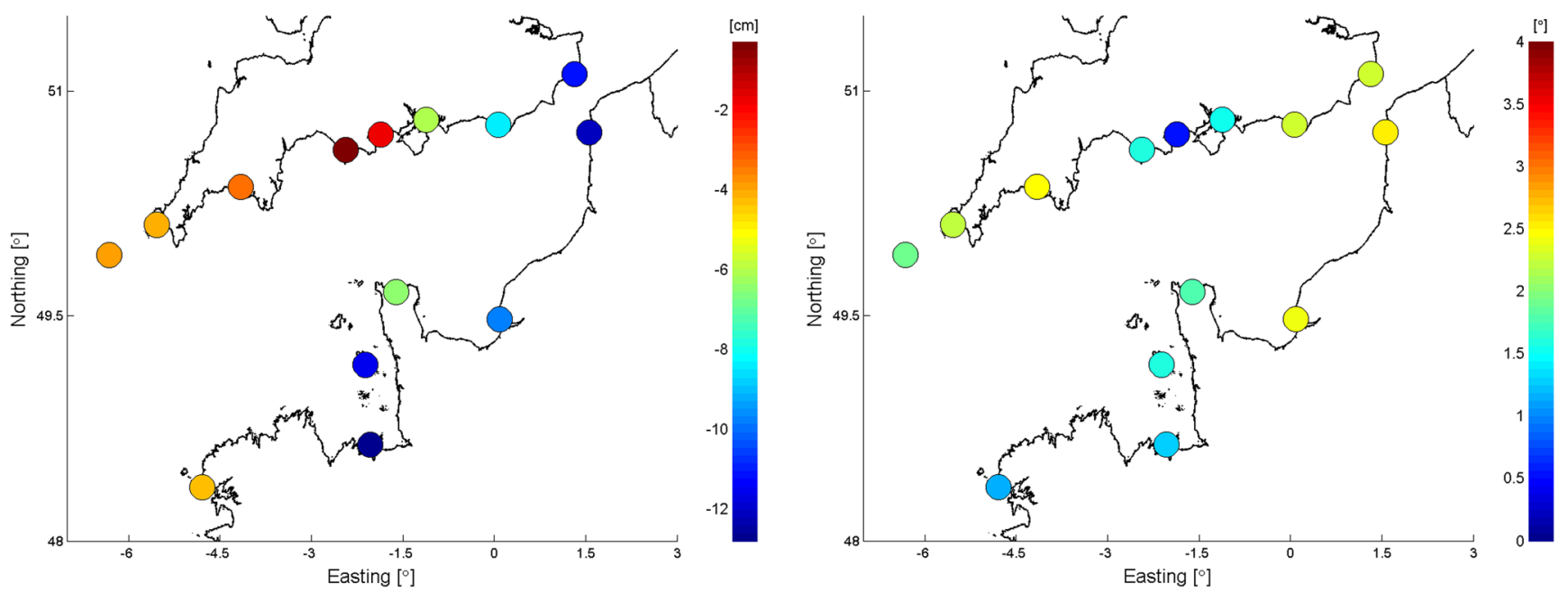

Fig. 7 Impact of SAL term in M2 amplitude $\left(H_{\mathrm{SAL}}-H_{\text {no SAL }}\right.$, left $)$ and phase $\left(G_{\mathrm{SAL}}-G_{\text {no SAL }}\right.$, right $)$ in observation stations in the English Channel 

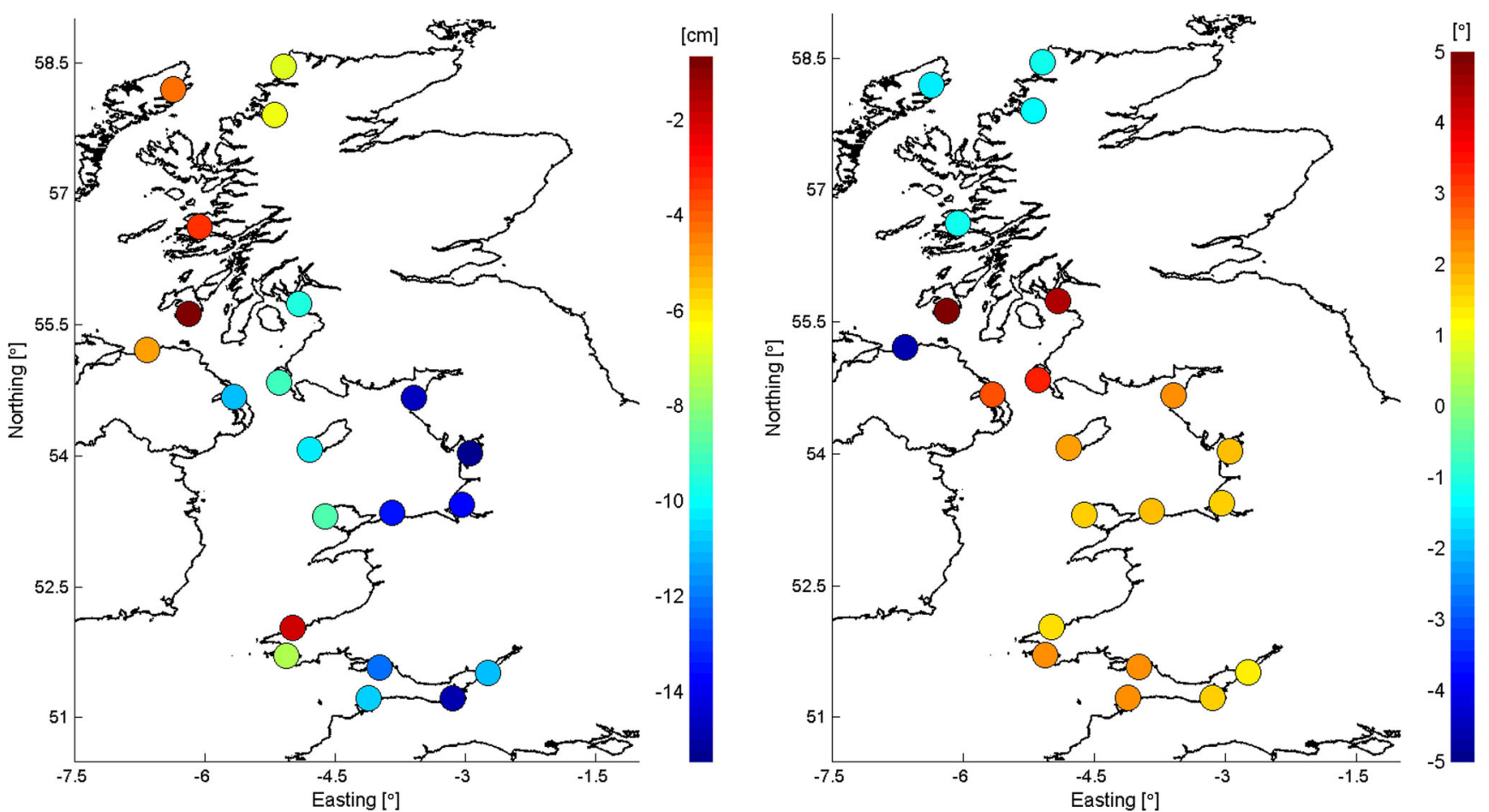

Fig. 8 Impact of SAL term in M2 amplitude $\left(H_{\mathrm{SAL}}-H_{\mathrm{no}} \mathrm{SAL}\right.$, left $)$ and phase $\left(G_{\mathrm{SAL}}-G_{\mathrm{noSAL}}\right.$, right $)$ in observation stations in the extended Irish Sea area

Fishguard up to Portrush, and only slightly underestimated in the remaining stations northwards. These drastic changes are due to the proximity to the amphidromic points in the southern (St. George's Channel) and northern entrances to the Irish Sea, as mentioned before. The phase increases due to SAL almost everywhere except for the stations in the very north of the Irish Sea (north of Port Ellen, in the west coast of Scotland). See Table 6 in Appendix B for results per station.

One can observe from Fig. 4 that there is also a relatively significant impact in $\mathrm{S} 2$ in the Irish Sea. This originates mainly from an impact on the phase error (from 8.3 to $6.9^{\circ}$ ), which improves remarkably in the stations in the east coast of the Irish Sea.

\subsubsection{Results for the North Sea}

We focus now on the impacts on stations over the North Sea. In the time domain, results show reduction of the total average RMSE of $2.3 \mathrm{~cm}$, and the error reduces in every station. Again, looking at the harmonic analysis results, the component that most improves and is the main contributor to the overall improvement of the tide RMSE is M2, with an average reduction of the vector difference of $4.9 \mathrm{~cm}$. Figure 9 shows the amplitude and phase errors similarly to previous figures for the whole set of observation stations in the North Sea.

Figure 9 shows a consistent direction of the impact of SAL across stations, with a reduction of amplitude and a slowdown of the M2 propagation. These impacts lead to an improvement of the total representation (VD) in every station, since without the SAL effect, the amplitudes where overestimated and phases where too low almost everywhere.

The stations north of the Calais Strait are strongly impacted by the SAL inclusion both in amplitude and in phase with vector difference changes reaching $15 \mathrm{~cm}$. The representation of the tide at these stations was remarkably poor in comparison with the rest of stations in the North Sea, and clearly benefit from the SAL effect. The east coast of the UK also shows a remarkable improvement, mainly in amplitudes.

M2 co-tidal charts constructed for the model domain have shown that the SAL has an effect on the location of the two amphidromic points in the North Sea (north of English Channel and west of Denmark) and translate into an impact in the east coast of the UK, Dutch Coast and German Bight, which result in better modelled tides. For the German Bight, in particular, SAL effects result in bigger amplitude errors, but the phase improves so much that the resulting VD reduces everywhere except for Cuxhaven. See Table 7 in Appendix $\mathrm{B}$ for results per station.

\subsubsection{Results for the Dutch Coast}

A more complete display of the results is included in this section, from time domain to frequency domain.

Table 1 shows the errors of the water levels in terms of RMS errors (time domain) for the runs without SAL and with SAL, for the stations in the Dutch Coast. The average RMS 

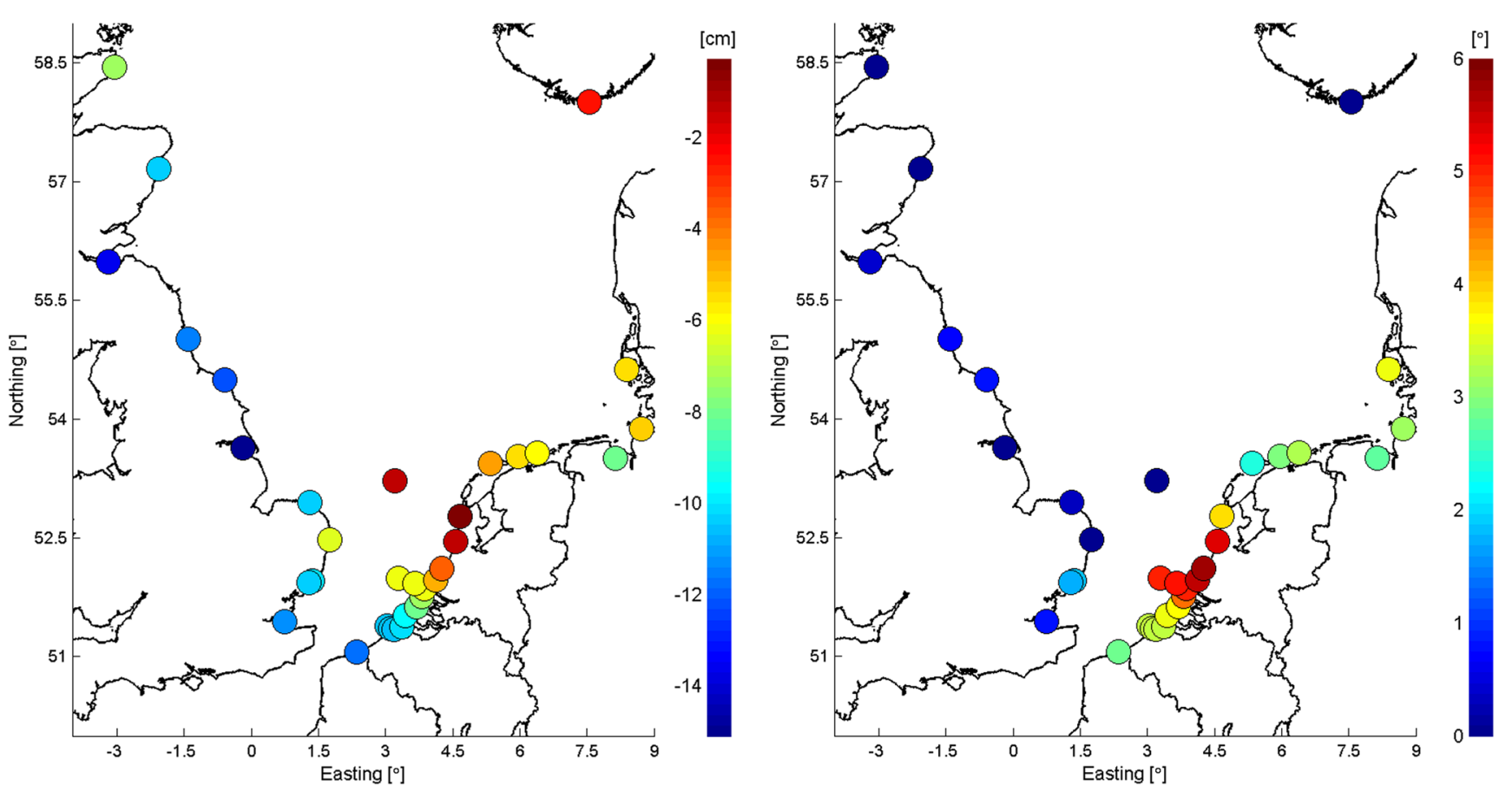

Fig. 9 Impact of SAL term in M2 amplitude $\left(H_{\mathrm{SAL}}-H_{\mathrm{no}} \mathrm{SAL}\right.$, left $)$ and phase $\left(G_{\mathrm{SAL}}-G_{\mathrm{no}} \mathrm{SAL}\right.$, right $)$ in observation stations in the North Sea

error in tidal signal reduces in $1 \mathrm{~cm}$. One can observe that, with the inclusion of the SAL term, the modelled tidal signal matches better observation everywhere along the Dutch Coast, except for Roompot buiten.

For a more insightful understanding of the SAL effect on propagation and modulation of the tides, we have a look at the modelled phases and amplitudes in comparison to the corresponding observed values. Table 2 shows the vector difference

Table 1 Impact of SAL in the tide RMSE (cm) for the Dutch Coast stations

\begin{tabular}{llll}
\hline Station & \multicolumn{2}{l}{ RMSE tide } & \multirow{2}{*}{ Difference } \\
\cline { 2 - 3 } & Without SAL & With SAL & \\
\hline Cadzand & 15.0 & 10.5 & -4.5 \\
Westkapelle & 13.9 & 10.2 & -3.7 \\
Roompot buiten & 11.6 & 13.8 & 2.2 \\
Brouwershav. Gat 08 & 12.3 & 9.7 & -2.6 \\
Haringvliet 10 & 13.5 & 9.8 & -3.7 \\
Hoek van Holland & 13.8 & 10.4 & -3.4 \\
Scheveningen & 12.0 & 10.0 & -2.0 \\
IJmuiden buitenhaven & 12.3 & 11.0 & -1.2 \\
Petten zuid & 11.5 & 11.0 & -0.5 \\
K13a platform & 7.5 & 7.1 & -0.4 \\
Terschelling Noordzee & 10.0 & 9.5 & -0.5 \\
Wierumergronden & 10.1 & 9.6 & -0.5 \\
Huibertgat & 11.7 & 10.5 & -1.3 \\
Total (RMS) & 12.1 & 10.3 & -1.8 \\
\hline
\end{tabular}

in centimetres for the five main constituents in the North Sea, including both diurnal and semi-diurnal species.

Results show that the main constituent that benefits from the inclusion of the SAL is M2, with a reduction of the vector difference of $5 \mathrm{~cm}$. For the rest of the components, the results do not change so much with or without SAL. Therefore, we take a closer look at M2 and look at its propagation and modulation throughout the different observation stations in the Dutch Coast.

Figure 10 shows the amplitude and phase impact in each of the stations considered due to SAL. The effect of the SAL term is as expected, i.e. it slows down propagation and reduces amplitudes. This happens consistently in all stations considered, except for the K13a platform, which shows a slight decrease in phase. The Dutch Coast shows a very positive impact of the addition of SAL to the model, with remarkably low amplitude and specially phase errors (in general below $1^{\circ}$ ). These results are excellent for an uncalibrated setup. See Table 8 in Appendix B for results per station.

Table 2 Impact of SAL per constituent for the Dutch Coast

\begin{tabular}{|c|c|c|c|c|c|c|}
\hline \multirow[t]{2}{*}{ Constituent } & \multicolumn{2}{|c|}{$\mathrm{RMS} \Delta H(\mathrm{~cm})$} & \multicolumn{2}{|c|}{$\operatorname{RMS} \Delta G\left(^{\circ}\right)$} & \multicolumn{2}{|c|}{ RMS VD (cm) } \\
\hline & No SAL & SAL & No SAL & SAL & No SAL & SAL \\
\hline $\mathrm{O} 1$ & 0.9 & 2.0 & 24.4 & 22.2 & 4.8 & 4.5 \\
\hline $\mathrm{K} 1$ & 1.0 & 0.9 & 22.4 & 25.7 & 3.4 & 3.8 \\
\hline $\mathrm{N} 2$ & 1.5 & 1.8 & 2.6 & 5.1 & 1.7 & 2.2 \\
\hline M2 & 6.2 & 3.2 & 4.0 & 1.5 & 9.6 & 4.6 \\
\hline S2 & 0.9 & 1.0 & 4.7 & 4.2 & 2.8 & 2.6 \\
\hline
\end{tabular}



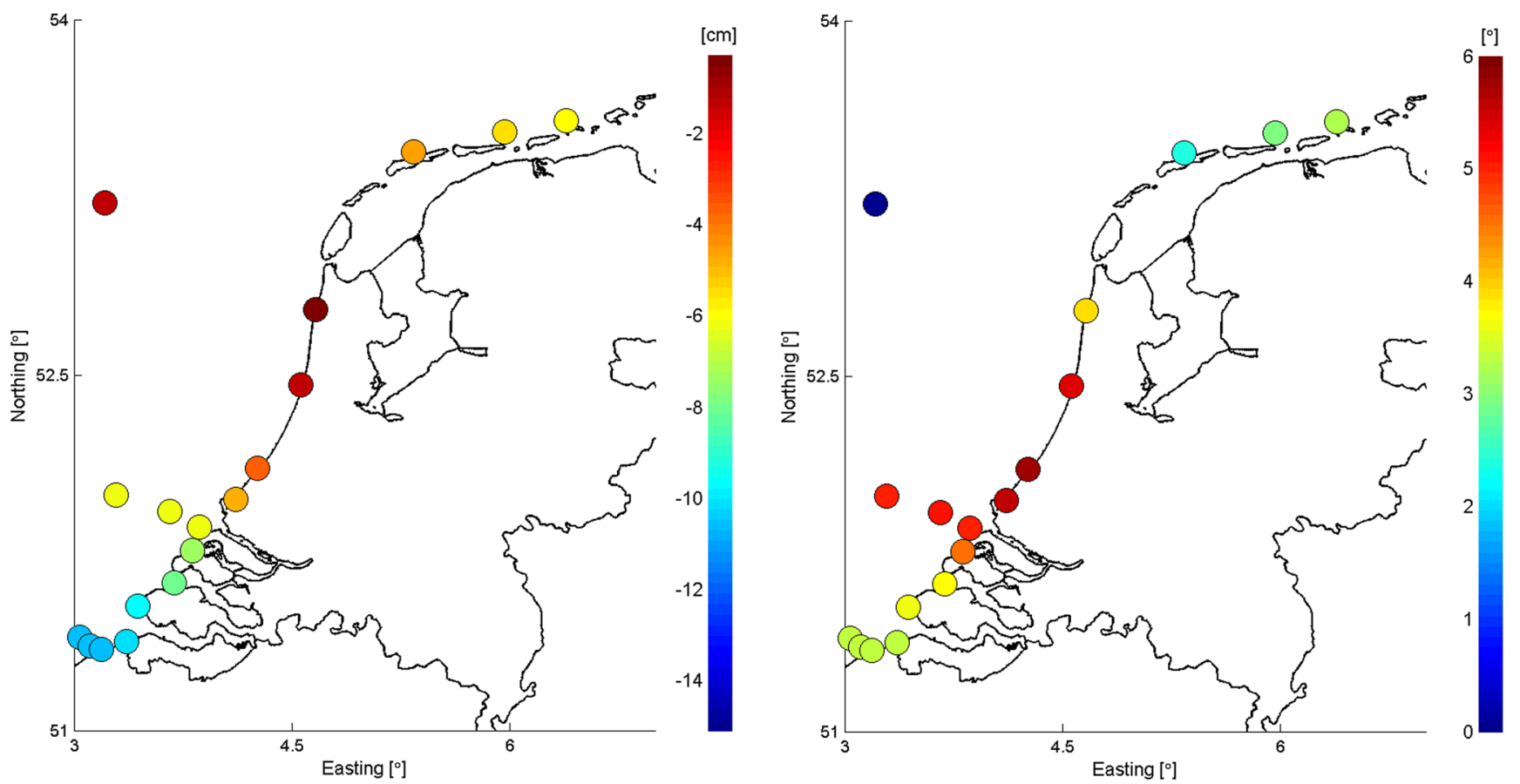

Fig. 10 Impact of SAL term in M2 amplitude $\left(H_{\mathrm{SAL}}-H_{\mathrm{no}} \mathrm{SAL}\right.$, left $)$ and phase $\left(G_{\mathrm{SAL}}-G_{\text {no SAL }}\right.$, right $)$ in observation stations in the Dutch Coast

\subsection{Comparison against beta approximation}

In this section, results using the beta approximation in comparison to results using the full SAL calculation are presented. This is done in the frequency domain for a better understanding of the differences in impact in the tidal representation between the two methods.

In this test, a constant value of beta is used for the whole domain. After a few iterations with values that can approximate the average beta according to Fig. 1, the value chosen is $0.015(1.5 \%)$. We remind the reader that the implementation of the beta parameterization used in this study is yet a simplification of the real parameterization given in Eq. (8) and should therefore be interpreted as such. This simplification consists on a reduction of gravity (see Eq. (9)), and it is therefore only applicable as a uniform reduction throughout the domain. A future study with an implementation of the beta parameterization as expressed in Eq. (8) and with a spatially variable field (see Fig. 1) is recommended. For the scope of this study and comparison, the used parameterization is deemed sufficient.

We now have a look at the average amplitude, phase and vector difference errors for M2 in the different areas (Table 3). With the use of a full SAL calculation, the phase errors reduce for every area and so do the amplitudes as well, except for the Irish Sea. For the beta approximation, phase error also improves everywhere almost as much as with the full SAL computation. For the amplitudes, however, the beta approximation leads to an increase of these everywhere expect for the Irish Sea.

For the Irish Sea, the amplitude error sign in the original model changes across stations, overestimating amplitudes in places and underestimating in others. This is due to the existence of two amphidromic points in a relatively narrow sea. Therefore, there is not a consistent improvement/worsening of the results when including SAL effects. More attention is
Table 3 Impact of SAL effect in M2 RMS amplitude error $(\Delta H)$, phase error $(\Delta G)$ and vector difference $(V D)$ per area in the model domain

\begin{tabular}{|c|c|c|c|c|c|c|c|c|c|}
\hline \multirow[t]{2}{*}{ Area } & \multicolumn{3}{|l|}{$\Delta H(\mathrm{~cm})$} & \multicolumn{3}{|l|}{$\Delta G\left(^{\circ}\right)$} & \multicolumn{3}{|l|}{$\mathrm{VD}(\mathrm{cm})$} \\
\hline & No SAL & $\beta$ & SAL & No SAL & $\beta$ & SAL & No SAL & $\beta$ & SAL \\
\hline English Channel & 8.8 & 12.1 & 5.9 & 5.2 & 4.4 & 3.5 & 22.8 & 20.4 & 15.2 \\
\hline Irish $\mathrm{Sea}^{\mathrm{a}}$ & 18.5 & 16.2 & 20.7 & 5.3 & 4.4 & 3.7 & 24.5 & 22.9 & 25.3 \\
\hline Dutch Coast & 6.2 & 8.0 & 3.2 & 4.0 & 1.5 & 1.5 & 9.6 & 8.7 & 4.6 \\
\hline North Sea & 9.9 & 11.1 & 5.2 & 5.4 & 3.9 & 4.2 & 16.6 & 15.4 & 11.7 \\
\hline $\mathrm{All}^{\mathrm{a}}$ & 16.0 & 15.9 & 15.9 & 8.2 & 8.4 & 8.0 & 23.5 & 22.8 & 21.9 \\
\hline
\end{tabular}

${ }^{\text {a }}$ Port Ellen excluded 
needed in this area, with potentially a finer resolution in the global model needed for the computation of the SAL potential.

\section{Discussion}

For all the areas, the SAL shows a fairly consistent impact, reducing amplitudes and increasing phases or, in other words, slowing down the M2 propagation. Since in most of the stations the results from the reference run show overprediction of amplitudes and too small phases (see Appendix B for a breakdown of all the stations), the inclusion of SAL results in an improvement of the M2 tide representation for almost every subdomain, as shown in Table 3 . The only outlier is the Irish Sea. This area shows to be very sensitive to the inclusion of SAL, with drastic changes in phase and amplitudes. This is associated to the existence of two amphidromic points in the area and very close to the observation stations due to the narrowness of the sea. The location and nature of these amphidromic points are eventually affected by the SAL term, as are the other two amphidromic points in the North Sea. The magnitude of the impact is clearly high, but the effect on the quality of the M2 representation is non-conclusive, improving for some stations and worsening for others, with an almost identical total vector difference.

It is noteworthy than the impact of the SAL is clearly nonnegligible, but the fact that the results improve with the addition of this term originates from the overprediction of amplitudes and propagation speeds in the base run, and therefore, the direction of the impact of the SAL results in an improvement. This initial overprediction can have other sources than the lack of SAL. For example, the used uniform value for bottom friction coefficient is somewhat trivial, only based on the typical range of values for Manning bottom friction coefficients for similar applications. The Manning formulation is known to give very good results in in coastal waters but results in an almost non-existent bottom drag in deeper waters, which might lead to an underestimation of the dissipation of barotropic energy in these regions and therefore too high amplitudes approaching the coastal waters. Moreover, the lack of other dissipative mechanisms like the internal tides drag in the shelf break of the regional model potentially also contributes to this overprediction of amplitudes. Although roughness is mainly known to influence amplitudes, it has also effects on phases, although to a much lesser extent. We therefore want to emphasize the impact magnitude of the SAL term rather than the resulting accuracy levels. Although these are also interesting, they are less conclusive due to the uncertainties in model settings of the base run. From the co-tidal charts generated for the case without and with SAL, we observe that the SAL term impacts slightly the location of amphidromic points and triggers changes in the phase and amplitude patterns.
As depicted by the regional tables in Appendix B, RMS differences for amplitude and phase and the corresponding bias show that the impact mainly results from a big impact in bias of both amplitude and phase errors in each region, and the standard deviation remains the same for most of the areas. This particularly holds for phase impacts. The only exception to this pattern is the Irish Sea, which does show also some impact on the standard deviation. For this area, however, uncertainties arising from the SAL field and possible local bottom friction effects make the interpretation of the impact difficult. For the amplitudes, statistics also show a dominant impact in bias, although in general, there is also a small contribution of the standard deviation to the amplitude error RMS. However, the bias varies significantly from region to region for both phase and amplitude, showing that the impact of SAL in somewhat uniform inside each area but shows an area-to-area spatial variability both in amplitude and phase.

In terms of the harmonic analysis performed, small errors on the derived harmonic constants might arise from the relatively short period used for the analysis. In a period of 2 months, it is not possible to capture lower frequency modulations with, for example, semi-annual or annual periodicity. For a more complete analysis, a longer period for the analysis is recommended in the future. Furthermore, in this study, only tidal representation at coastal stations against in situ measurements is compared. A comparison with satellite altimetry data for a spatially well-distributed coverage of the domain is recommended in order to understand well the SAL spatial impact on accuracy of tidal representation also offshore and in deep waters.

The methodology followed in this paper using the SAL field calculated from the GTSM as surface forcing in the regional model also has some limitations. Firstly, inconsistencies are expected between the global SAL tide and the water levels from the regional model. The regional model solution is strongly constrained by the water level forcing at the open boundaries, obtained from an assimilative global model (FES2012). In the non-assimilative GTSM global model, however, the solution in the domain of the regional model is determined by the tidal wave propagating throughout the whole world, which has been verified in a global scale but has not been calibrated for accuracy levels comparable to FES2012 in the Northwest European Continental Shelf. Errors in remote areas of the world in M2 propagation are expected to influence local results, and the model has not as of yet undergone a region-tailored calibration. Secondly, the SAL potential is calculated in the GTSM model in a $1^{\circ}$ resolution grid, which for global effects seems to be enough but might not be sufficient to accurately represent regional scale SAL effects, especially in narrow semi-enclosed seas. An example of this is the Irish Sea, in which this resolution is clearly insufficient to represent the gradients originating from the presence of the two amphidromic points. Inconsistencies of 
Table 4 Average tide RMS error (time domain) for each area in the model domain for the cases without SAL, with beta approximation and with full SAL calculation

\begin{tabular}{llll}
\hline \multirow{2}{*}{ Area } & \multicolumn{2}{l}{ RMSE tide $(\mathrm{cm})$} & \\
\cline { 2 - 4 } & Without SAL & With $\beta$ & With full SAL \\
\hline English Channel & 20.0 & 18.2 & 15.5 \\
Irish Sea $^{\mathrm{a}}$ & 27.2 & 26.7 & 26.9 \\
Dutch Coast $_{\text {North Sea }}$ & 12.1 & 12.0 & 10.3 \\
All $^{\mathrm{a}}$ & 16.5 & 15.9 & 13.9 \\
\hline
\end{tabular}

${ }^{\text {a }}$ Port Ellen excluded

the direction of the SAL impact in this area might be associated to this issue.

The comparison of results from a full spherical harmonicbased SAL computation and the (simplified) beta approximation show an equivalent RMS impact in phases per area, but an opposite direction of impact in amplitudes. It has been shown in the station-based results that the general impact of SAL is translated into a reduction of amplitudes and a slowdown of propagation at coastal stations, and therefore including SAL improves the M2 tidal representation when compared to the overpredicted amplitudes and propagation speeds of the base run without SAL. Reducing the barotropic pressure gradient through the beta coefficient slows down the propagation, but it also increases amplitudes, which impacts negatively the already overestimated amplitudes. This penalizes the resulting vector difference which, although lower than the reference run, is not as low as for the full SAL computation. Additionally, in a station by station analysis, the results from the two methodologies differ significantly. In total terms, though, the beta approximation leads to an improved modelled M2 in comparison with the case without SAL. In this study, a uniform value for the $\beta$ coefficient has been used for simplicity, but the optimal value of this parameter has proved to be strongly spatially varying in this continental shelf domain. Therefore, the interpretability of the results from the comparison with a full SAL computation is limited, but it gives an idea for the direction of the impact through the beta approach and makes evident the difference between the two on the impact direction for the amplitudes. These optimal $\beta$ values are expected to change when including different (physical) processes, forcings or when making changes to the geometry or model settings, and therefore, recalibration of this parameter would be needed upon any similar changes. Moreover, the remarkable differences between the optimal $\beta$ for the SAL effect originating from the tidal motion and the $\beta$ field associated to other loads that also trigger SAL effects such as the ocean circulation (see Fig. 2.a in Kuhlmann et al. (2011)) or atmospheric forcing (see Fig. 3c in Stepanov and Hughes (2004)) shows the incapability of the beta approximation to accommodate the effects due to combined SAL sources while maintaining an acceptable correlation level. It is concluded from this that, if one wants to account for SAL effects from different sources (periodic and non-periodic), an online, time-stepped and spatially integrated calculation of the SAL field seems unavoidable already at a global scale.

Although in this study we have focused on the impact on tide, and this has proved to be significant, further research is needed to assess the potential impact on the modelled surge indirectly from non-linear tide-surge interaction. Additionally, other loads like meteorological forcing and hydrology can also be expected to have an impact in a regional scale. In particular for the meteorological forcing, literature shows that the correlation between the associated SAL and the total water levels is remarkably poor even at global scales. In order to accurately include the SAL effects originating from nonperiodic motions like surges, the full SAL calculation would need to be integrated directly in the momentum equations in the regional model. This poses a challenge, due to the need to know the instantaneous water level solution throughout the world to compute the instantaneous SAL effect locally.

We have shown that the effects of the self-attraction and loading in tidal propagation in a regional scale are not negligible when compared to the accuracy order of magnitude that regional barotropic tide surge models are currently aiming for. Furthermore, including this physical process in an uncalibrated regional model results in an almost spatially consistent improvement of the tidal representation, especially in terms of phase, although further investigation of the origin of initially overpredicted amplitudes and propagation speeds is needed to get a clear conclusion in this respect. For barotropic tide surge models, it is common practice to calibrate bathymetry in order to correct the phase errors and get propagation speeds correct, since depth is the most determining parameter for the propagation speed. A calibration of the bottom friction coefficient usually follows this to compensate for amplitude errors. The remarkable phase error reduction throughout the domain due to SAL looks appealing to minimize bathymetry adjustments in the calibration phase, since the bathymetry is not a user-specified parameter part of a parameterization (unlike the bottom friction coefficient) but a real feature of the domain, despite the possible uncertainties of the bathymetry data. This has the potential to lead to more realistic models and to a less calibration-dependent reliability of the models. It would be interesting to find out what are the implications of the addition of the SAL term on the subsequent calibration of the regional mod$\mathrm{el}$, and the impact of the addition of other processes that affect tidal propagation such as the generation of internal tides at the shelf break on the SAL effects. 


\section{Summary and conclusions}

In this study, the impact of the inclusion of the selfattraction and loading effect (SAL) in a regional 2D barotropic tidal model has been assessed, a term with acknowledged importance and well understood for global models but omitted for boundary-forced, regional models. We have forced a regional model for the Northwest European Shelf and North Sea (CSM) with the SAL potential field derived from a global model (GTSM), in the form of a pressure field. Impacts have been studied in an uncalibrated setup for CSM and with only tidal forcing on, in order to isolate effects. Additionally, the usually accepted so-called beta approximation, in which the SAL effect is parameterized as a percentage of the barotropic pressure (typically chosen $10 \%$ ), is also implemented in a simplified manner and compared to the results obtained with a full SAL computation.

The derivation of the optimal beta coefficient spatially for the whole globe results indeed in a value of $\sim 10 \%$ for deep waters (consistent with literature) but gives very different values for shallow waters in several areas of the world. Based on this computed beta field and its values for the North West Continental Shelf, an average value range for the North Sea area of around $1.5-5 \%$ is concluded and tested for the beta approximation approach, using a simplified parameterization. The correlation between the SAL tide and an water level is also computed, showing a noteworthy poor correlation for several shelf areas like the North Sea.

The period simulated is from January 2007 to March 2007, long enough for making use of harmonic analysis on the time series. Results are compared to coastal tide gauge data of representative stations throughout the whole domain, with a special attention in the coast of the Netherlands, due to the importance of this model for the country's operational flood forecasting. The results are presented in terms of RMS errors in the time domain and further expanded in the frequency domain to look at impacts on individual constituents and separate impacts in amplitude and phase.

Firstly, the impact of the inclusion of SAL in the co-tidal charts resulting from a harmonic analysis at each grid node in the domain is presented. This provides a better understanding of the spatial distribution and intensity of the impact. The biggest impact of M2 is observed in the English Channel, Irish Sea and the West (UK East coast) and South (Belgian and Dutch Coast) of the North Sea. Impact reaches values of $20 \mathrm{~cm}$ in terms of vector difference relative to the reference run without SAL. Other constituents like S2 also show an impact that can reach values of $4 \mathrm{~cm}$. For simplicity, we focus on M2 for an analysis of the tidal representation in the observation stations of the impacted areas in the frequency domain.

The impact of SAL translates into a consistent M2 amplitude and propagation speed reduction throughout the domain. This leads to a reduction of errors in the tidal representation due to the initially overpredicted amplitudes and propagation speeds in the reference run without SAL. An exception is the Irish Sea, inside which interpretation of the SAL effect is difficult due to possible lack of resolution of the SAL field, existence of two amphidromic points locally and possible local bottom friction effects were not accounted for in this uncalibrated setup. In the time domain, the average RMS error reduces for all the areas, as presented in Table 4.

Results using the (simplified) beta approximation, with a value of $1.5 \%$, are compared against the reference run and full SAL computation run results. An analysis in frequency domain of the resulting M2 amplitudes and phases for the two implementations shows that in terms of phase, both implementations have a comparable impact. In terms of amplitudes, the beta approximation shows an opposite direction of the impact, increasing amplitudes everywhere. In terms of quality of the results, the beta approximation method shows better results in all the subdomains than the reference, SAL lacking run (see Table 3), although the full computation of SAL shows further improved results everywhere except in the Irish Sea, for which results are comparable. Again, this quality assessment is based on a reference, SAL lacking run with overpredicted amplitudes and underpredicted phases. We therefore conclude that the beta approximation is not enough to represent the effects of the real SAL in space and time.

After showing evidence of the SAL impact in regional models, the question of how to include this physical process in them in an efficient way arises. Since SAL is a non-local effect and depends on the instantaneous water levels over the whole ocean, this task is non-trivial. In this case, as a short-term fit to purpose solution, the output of a global model has been used to force a regional model, but this does not constitute a viable methodology for simulations of longer periods and less so for operational forecasting. A solution for SAL originating from tide is presented by Teng et al. (2016), who use pre-computed SAL co-tidal charts from a global model to synthesize the total SAL tide from its harmonic components and include it at each time step. This can be a solution for the SAL effects originating from the astronomical tidal forcing, but does not hold for other non-periodic loads. The beta approximation, although simple and useful in an only-tide run, is constrained by the same limitations, since very different beta fields are associated to the different possible loads. 


\section{Appendix A Location of observation stations}

Fig. 11 Set of observation stations used for the comparison with modelled water levels. Red: Irish Sea (extended

southwards).Green: North Sea. Black: estuarine stations in the Dutch Coast, included in the statistics of all the stations. Blue: English Channel

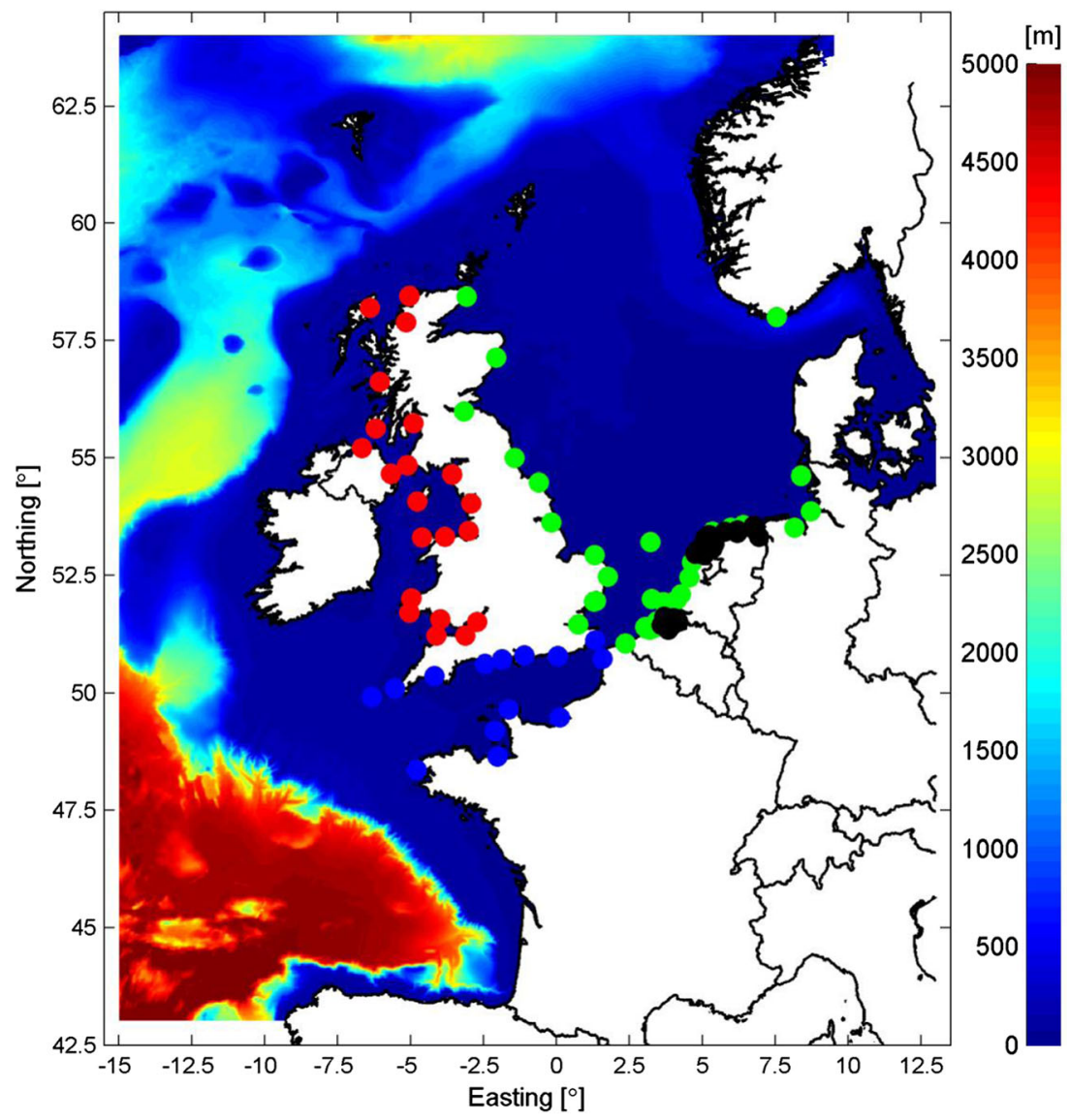

Numbering ID for stations: 1. Wick; 2. Aberdeen; 3 . Leith; 4. Northshields; 5. Whitby; 6. Immingham; 7. Cromer; 8. Lowestoft; 9. Felixstowe; 10. Harwich; 11. Sheerness; 12.Dunkerque; 13. Wandelaar; 14. A2; 15. Zeebrugge; 16. Cadzand; 17. Westkapelle; 18. Europlatform; 19 . Roompot buiten; 20 . Brouwershavensche Gat 08; 21. Haringvliet 10; 22. Licheiland Goeree; 23. Hoek van Holland; 24. Scheveningen; 25. IJmuiden buitenhaven; 26. K13a platform; 27. Petten zuid; 28. Terschelling Noordzee; 29. Wierumergronden; 30 . Huibertgat; 31. Wilhelmshaven; 32. Cuxhaven; 33. Wittduen; 34. Tregde; 35. St. Mary’s; 36. Newlyn; 37.Le Conquet; 38. Devonport; 39. Weymouth; 40. Saint Malo; 41. St. Helier, Jersey; 42. Cherbourg; 43. Bournemouth; 44.
Portsmouth; 45. Newhaven; 46. Le Havre; 47. Dover; 48. Boulogne Sur Mer; 49. Milford; 50. Ilfracombe; 51. Mumbles,; 52. Hinkley Point; 53. Avonmouth; 54. Fishguard; 55. Holyhead; 56. Llandudno; 57. Liverpool; 58. Heysham; 59. Port Erin; 60.Workington; 61. Portpatrick; 62. Bangor; 63. Millport; 64. Portrush; 65. Port Ellen; 66. Tobermory; 67. Ullapool; 68. Stornoway; 69. Kinlochbervie; 70. Vlissingen; 71. Terneuzen; 72. Roompot binnen; 73. Stavenisse; 74. Bergse Diepsluis west; 75. Den Helder; 76. Oudeschild; 77. Den Oever buiten; 78. Vlieland haven; 79. West-Terschelling; 80 . Kornwerderzand buiten; 81. Harlingen; 82. Nes; 83. Lauwersoog; 84. Schiermonnikoog; 85. Borkum; 86. Eemshaven; 87. Delfzijl 
a

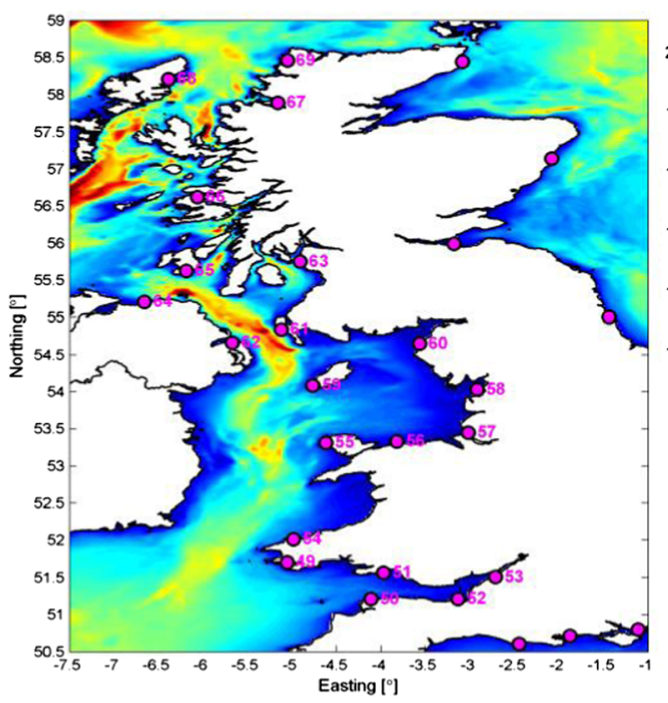

C
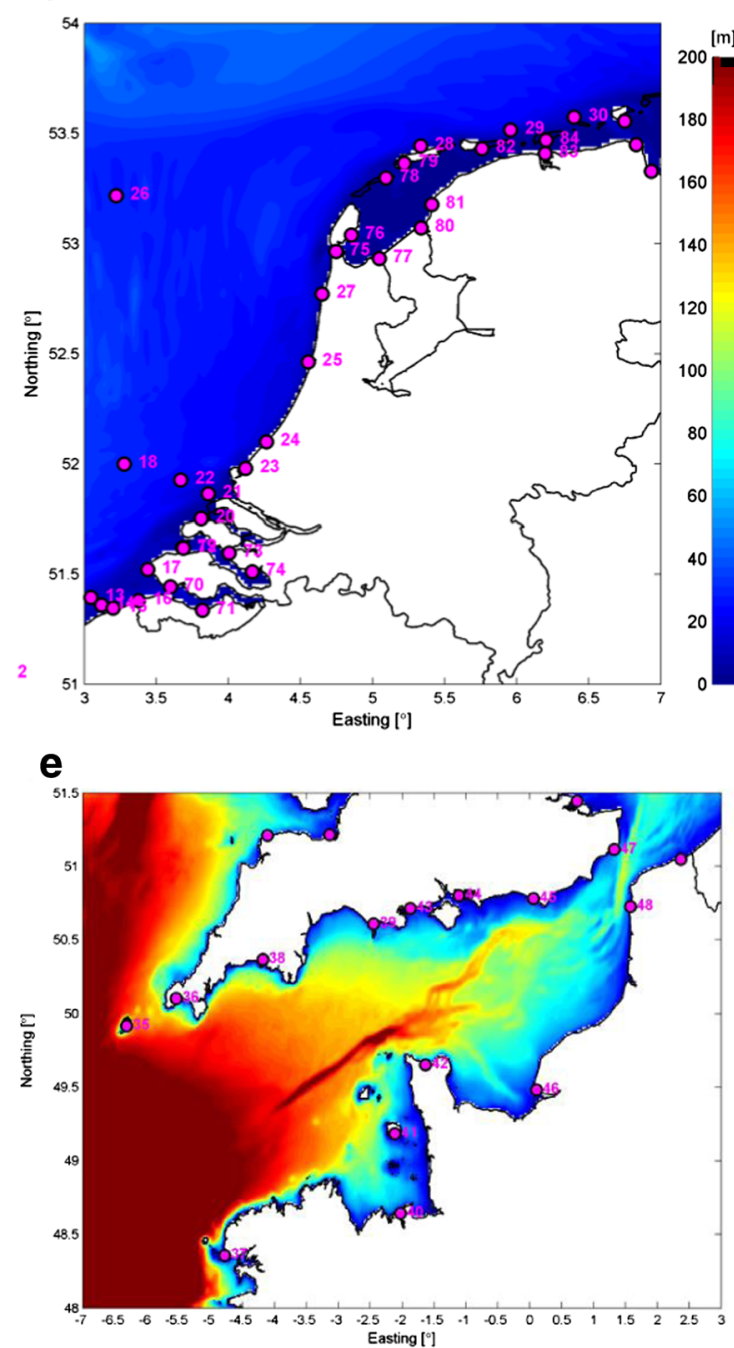

Fig. 12 Detail for the different areas, with corresponding station numbers. a Station of the extended Irish Sea area. b Detail of stations at the UK East coast from North Sea area. $\mathbf{c}$ Stations of the Dutch Coast area b

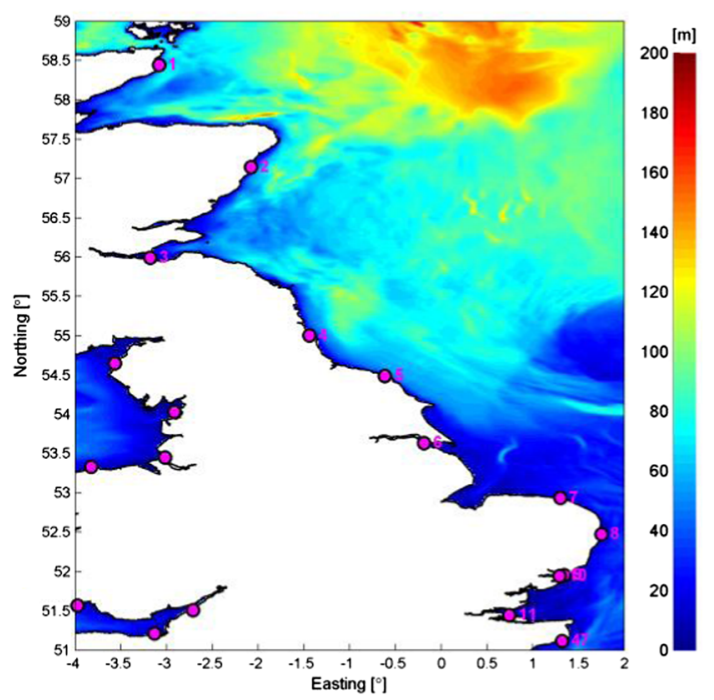

d

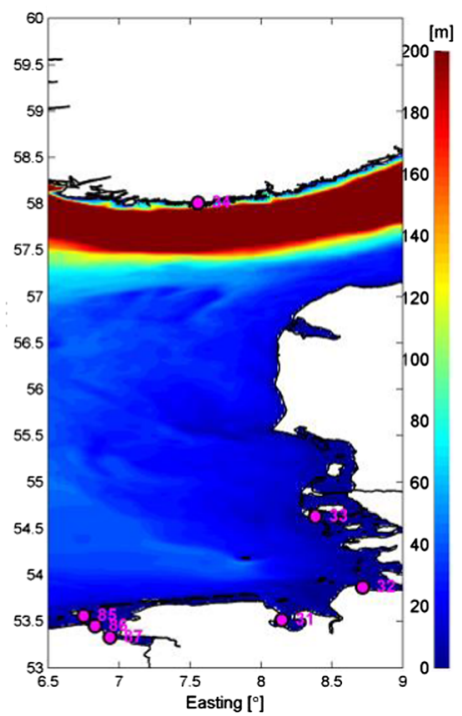

and estuarine stations. d Detail of the stations in the German Bight. e Detail of the stations in the English Channel 


\section{Appendix B SAL impact on M2 per station}

Table 5 Impact of SAL effect in M2 amplitude error $(\Delta H)$, phase error $(\Delta G)$ and vector difference (VD) per station in the English Channel

\begin{tabular}{|c|c|c|c|c|c|c|c|c|c|c|c|c|}
\hline \multirow[t]{2}{*}{ Station } & \multirow[t]{2}{*}{ St. no. } & \multirow[t]{2}{*}{$H_{\mathrm{o}}$} & \multirow[t]{2}{*}{$G_{\mathrm{o}}$} & \multicolumn{3}{|l|}{$\Delta H(\mathrm{~cm})$} & \multicolumn{3}{|l|}{$\Delta G\left(^{\circ}\right)$} & \multicolumn{3}{|l|}{$\mathrm{VD}(\mathrm{cm})$} \\
\hline & & & & No SAL & SAL & $\Delta$ & No SAL & SAL & $\Delta$ & No SAL & SAL & $\Delta$ \\
\hline St. Mary's & 35 & 176.2 & 130.9 & -3.0 & -6.9 & -3.9 & -3.2 & -1.4 & 1.9 & 10.3 & 8.0 & -2.3 \\
\hline Newlyn & 36 & 172.2 & 132.6 & 1.4 & -2.7 & -4.1 & -3.9 & -1.7 & 2.2 & 11.9 & 5.7 & -6.2 \\
\hline Le Conquet & 37 & 201.9 & 109.6 & 0.5 & -3.8 & -4.3 & -1.1 & 0.1 & 1.2 & 3.8 & 3.8 & -0.0 \\
\hline Devon port & 38 & 168.3 & 152.7 & -2.0 & -5.4 & -3.4 & -8.9 & -6.4 & 2.5 & 25.9 & 19.3 & -6.6 \\
\hline Weymouth & 39 & 57.8 & 189.3 & 1.1 & 0.8 & -0.3 & -5.2 & -3.6 & 1.6 & 5.4 & 3.7 & -1.7 \\
\hline Saint Malo & 40 & 364.8 & 176.4 & 1.4 & -11.4 & -12.8 & -7.1 & -5.8 & 1.3 & 45.3 & 38.1 & -7.3 \\
\hline St. Helier & 41 & 330.7 & 180.4 & 2.1 & -9.4 & -11.5 & -4.4 & -2.8 & 1.6 & 25.8 & 18.7 & -7.1 \\
\hline Cherbourg & 42 & 185.6 & 226.9 & 6.7 & 0.1 & -6.5 & -3.1 & -1.3 & 1.8 & 12.2 & 4.3 & -7.9 \\
\hline Bournemouth & 43 & 40.6 & 274.6 & 3.9 & 2.2 & -1.7 & -6.0 & -5.5 & 0.5 & 5.9 & 4.5 & -1.4 \\
\hline Portsmouth & 44 & 142.3 & 326.4 & 12.5 & 6.5 & -6.0 & -4.5 & -3.0 & 1.5 & 17.2 & 10.0 & -7.2 \\
\hline Newhaven & 45 & 228.3 & 320.8 & 5.0 & -3.4 & -8.4 & -6.0 & -3.7 & 2.3 & 24.8 & 15.2 & -9.6 \\
\hline Le Havre & 46 & 259.4 & 284.9 & 11.7 & 2.0 & -9.7 & -5.7 & -3.2 & 2.4 & 28.7 & 14.8 & -13.9 \\
\hline Dover & 47 & 228.1 & 330.2 & 21.6 & 10.4 & -11.2 & -4.0 & -1.7 & 2.3 & 27.2 & 12.5 & -14.7 \\
\hline Boulogne Sur Mer & 48 & 294.4 & 328.1 & 14.6 & 2.4 & -12.2 & -5.1 & -2.6 & 2.5 & 30.6 & 13.4 & -17.2 \\
\hline RMS & - & 222.0 & 239.6 & 8.8 & 5.9 & -2.8 & 5.2 & 3.5 & -1.7 & 22.8 & 15.2 & -7.6 \\
\hline Bias & - & - & - & 5.5 & -1.3 & -6.9 & -4.9 & -3.0 & 1.8 & - & - & - \\
\hline
\end{tabular}

Difference (SAL-no SAL) for each parameter is given by $\Delta$

Table 6 Impact of SAL effect in M2 amplitude error $(\Delta H)$, phase error $(\Delta G)$ and vector difference (VD) per station in the Irish Sea

\begin{tabular}{|c|c|c|c|c|c|c|c|c|c|c|c|c|}
\hline \multirow[t]{2}{*}{ Station } & \multirow[t]{2}{*}{ St. no. } & \multirow[t]{2}{*}{$H_{\mathrm{o}}$} & \multirow[t]{2}{*}{$G_{\mathrm{o}}$} & \multicolumn{3}{|l|}{$\Delta H(\mathrm{~cm})$} & \multicolumn{3}{|l|}{$\Delta G\left(^{\circ}\right)$} & \multicolumn{3}{|l|}{$\mathrm{VD}(\mathrm{cm})$} \\
\hline & & & & No SAL & SAL & $\Delta$ & No SAL & SAL & $\Delta$ & No SAL & SAL & $\Delta$ \\
\hline Milford & 49 & 221.8 & 172.0 & -7.2 & -14.7 & -7.6 & -4.4 & -2.2 & 2.3 & 18.2 & 16.8 & -1.4 \\
\hline Ilfracombe & 50 & 306.5 & 160.7 & -10.7 & -21.5 & -10.8 & -3.5 & -1.3 & 2.2 & 21.2 & 22.5 & 1.2 \\
\hline Mumbles & 51 & 314.9 & 165.0 & -11.5 & -23.6 & -12.0 & 1.3 & 3.5 & 2.2 & 13.5 & 30.1 & 16.6 \\
\hline Hinkley Point & 52 & 396.5 & 180.3 & -13.6 & -28.4 & -14.8 & -2.4 & -0.7 & 1.7 & 21.3 & 28.8 & 7.6 \\
\hline Avonmouth & 53 & 433.3 & 197.0 & -67.7 & -78.6 & -11.0 & 5.8 & 7.1 & 1.3 & 78.8 & 92.5 & 13.7 \\
\hline Fishguard & 54 & 133.8 & 206.7 & -9.6 & -11.6 & -2.0 & -2.1 & -0.6 & 1.5 & 10.7 & 11.7 & 1.0 \\
\hline Holyhead & 55 & 182.0 & 291.7 & 9.7 & 0.9 & -8.9 & -0.6 & 1.0 & 1.6 & 9.9 & 3.2 & -6.7 \\
\hline Llandudno & 56 & 271.2 & 308.8 & 15.3 & 1.8 & -13.5 & -1.2 & 0.5 & 1.8 & 16.4 & 3.1 & -13.3 \\
\hline Liverpool & 57 & 305.4 & 320.3 & 11.1 & -2.6 & -13.8 & -4.0 & -2.3 & 1.7 & 24.4 & 12.7 & -11.7 \\
\hline Heysham & 58 & 317.8 & 325.2 & 14.7 & -0.8 & -15.5 & 1.9 & 3.7 & 1.8 & 18.3 & 20.8 & 2.5 \\
\hline Port Erin & 59 & 184.7 & 322.3 & 19.2 & 8.9 & -10.3 & -3.0 & -0.8 & 2.2 & 21.7 & 9.3 & -12.4 \\
\hline Workington & 60 & 275.5 & 331.1 & 14.6 & -0.1 & -14.7 & -1.9 & 0.4 & 2.3 & 17.4 & 1.7 & -15.7 \\
\hline Portpatrick & 61 & 136.1 & 332.1 & 7.1 & -1.9 & -9.0 & -8.1 & -4.7 & 3.4 & 20.9 & 11.3 & -9.6 \\
\hline Bangor & 62 & 118.5 & 317.5 & 15.4 & 4.5 & -10.9 & -9.2 & -6.3 & 2.9 & 25.4 & 14.0 & -11.4 \\
\hline Millport & 63 & 114.0 & 342.4 & 4.8 & -4.6 & -9.5 & -14.0 & -9.5 & 4.5 & 28.7 & 19.0 & -9.7 \\
\hline Portrush & 64 & 52.4 & 198.3 & 10.1 & 5.1 & -5.0 & 9.5 & 4.9 & -4.6 & 13.9 & 7.0 & -6.9 \\
\hline Port Ellen & 65 & 16.1 & 84.5 & -12.5 & -13.2 & -0.7 & -176.0 & 36.7 & 212.8 & 19.7 & 13.9 & -5.8 \\
\hline Tobermory & 66 & 128.1 & 167.9 & -1.2 & -4.6 & -3.4 & -1.8 & -3.0 & -1.2 & 4.2 & 8.0 & 3.8 \\
\hline Ullapool & 67 & 149.8 & 200.3 & 2.0 & -4.5 & -6.5 & 0.3 & -1.0 & -1.3 & 2.1 & 5.2 & 3.1 \\
\hline Stornoway & 68 & 138.4 & 197.2 & -0.5 & -4.6 & -4.1 & 0.8 & -0.7 & -1.5 & 2.1 & 4.9 & 2.8 \\
\hline Kinlochbervie & 69 & 143.0 & 207.4 & 2.9 & -3.9 & -6.8 & 1.3 & 0.1 & -1.2 & 4.3 & 3.9 & -0.3 \\
\hline $\mathrm{RMS}^{\mathrm{a}}$ & - & 239.1 & 256.6 & 18.5 & 20.7 & 2.3 & 5.3 & 3.7 & -1.5 & 24.5 & 25.3 & 0.8 \\
\hline $\operatorname{Bias}^{\mathrm{a}}$ & - & - & - & 0.2 & -9.3 & -9.5 & -1.8 & -0.6 & 1.2 & - & - & - \\
\hline
\end{tabular}

Difference (SAL-no SAL) for each parameter is given by $\Delta$

${ }^{a}$ Port Ellen excluded 
Table 7 Impact of SAL effect in M2 amplitude error $(\Delta H)$, phase error $(\Delta G)$ and vector difference (VD) per station in the North Sea

\begin{tabular}{|c|c|c|c|c|c|c|c|c|c|c|c|c|}
\hline \multirow[t]{2}{*}{ Station } & \multirow[t]{2}{*}{ St. no. } & \multirow[t]{2}{*}{$H_{\mathrm{o}}$} & \multirow[t]{2}{*}{$G_{\mathrm{o}}$} & \multicolumn{3}{|l|}{$\Delta H(\mathrm{~cm})$} & \multicolumn{3}{|l|}{$\Delta G\left(^{\circ}\right)$} & \multicolumn{3}{|l|}{$\mathrm{VD}(\mathrm{cm})$} \\
\hline & & & & No SAL & SAL & $\Delta$ & No SAL & SAL & $\Delta$ & No SAL & SAL & $\Delta$ \\
\hline Wick & 1 & 101.0 & 321.3 & 15.8 & 8.4 & -7.4 & -4.4 & -6.4 & -2.1 & 17.8 & 14.5 & -3.3 \\
\hline Aberdeen & 2 & 129.1 & 24.1 & 13.5 & 3.2 & -10.2 & -2.5 & -2.5 & 0.0 & 14.7 & 6.5 & -8.2 \\
\hline Leith & 3 & 178.1 & 54.7 & 14.3 & 0.7 & -13.6 & -2.2 & -1.8 & 0.4 & 16.0 & 5.7 & -10.3 \\
\hline Northshields & 4 & 161.0 & 88.8 & 13.4 & 2.0 & -11.4 & -3.6 & -2.8 & 0.7 & 17.0 & 8.3 & -8.7 \\
\hline Whitby & 5 & 165.2 & 103.8 & 15.5 & 3.4 & -12.1 & -1.5 & -0.7 & 0.8 & 16.2 & 3.9 & -12.3 \\
\hline Immingham & 6 & 226.9 & 160.3 & 13.5 & -1.6 & -15.1 & -7.0 & -7.0 & -0.1 & 31.5 & 27.7 & -3.7 \\
\hline Cromer & 7 & 156.9 & 187.4 & 8.4 & -2.0 & -10.4 & 0.3 & 0.6 & 0.4 & 8.5 & 2.6 & -5.8 \\
\hline Lowestoft & 8 & 69.7 & 259.1 & 6.0 & -0.5 & -6.5 & -2.5 & -2.7 & -0.2 & 6.8 & 3.3 & -3.5 \\
\hline Felixstowe & 9 & 128.7 & 318.5 & 9.5 & -0.6 & -10.1 & -0.2 & 1.6 & 1.8 & 9.5 & 3.7 & -5.8 \\
\hline Harwich & 10 & 135.9 & 321.3 & 6.5 & -3.8 & -10.3 & -1.4 & 0.3 & 1.8 & 7.4 & 3.9 & -3.5 \\
\hline Sheerness & 11 & 204.6 & 350.8 & 2.1 & -9.2 & -11.4 & 7.7 & 8.5 & 0.8 & 27.6 & 31.0 & 3.4 \\
\hline Dunkerque & 12 & 216.8 & 350.2 & 13.1 & 1.3 & -11.7 & -4.5 & -1.6 & 2.9 & 21.8 & 6.2 & -15.6 \\
\hline Wandelaar & 13 & 160.1 & 8.9 & 17.8 & 7.2 & -10.6 & -5.9 & -2.5 & 3.4 & 24.8 & 10.1 & -14.7 \\
\hline A2 & 14 & 165.1 & 8.8 & 15.1 & 4.5 & -10.6 & -4.2 & -0.8 & 3.4 & 19.6 & 5.1 & -14.5 \\
\hline Zeebrugge & 15 & 164.8 & 11.3 & 15.8 & 5.3 & -10.6 & -5.3 & -2.0 & 3.4 & 22.6 & 7.8 & -14.7 \\
\hline Cadzand & 16 & 166.3 & 17.6 & 8.5 & -1.6 & -10.1 & -4.2 & -0.9 & 3.3 & 15.2 & 3.1 & -12.1 \\
\hline Westkapelle & 17 & 153.5 & 21.8 & 7.7 & -1.9 & -9.6 & -4.0 & -0.4 & 3.6 & 13.4 & 2.3 & -11.2 \\
\hline Europlatform & 18 & 74.9 & 22.5 & 9.5 & 3.5 & -6.1 & -2.1 & 2.9 & 5.0 & 10.0 & 5.2 & -4.8 \\
\hline Roompot buiten & 19 & 136.1 & 32.5 & 4.0 & -4.0 & -7.9 & 0.7 & 4.5 & 3.7 & 4.3 & 11.2 & 6.9 \\
\hline Brouwershav. Gat 08 & 20 & 111.8 & 36.5 & 7.8 & 0.5 & -7.3 & -3.6 & 1.0 & 4.6 & 10.7 & 1.9 & -8.7 \\
\hline Haringvliet 10 & 21 & 96.0 & 42.0 & 10.8 & 4.5 & -6.3 & -4.5 & 0.5 & 5.0 & 13.4 & 4.6 & -8.8 \\
\hline Licheiland Goeree & 22 & 87.7 & 36.5 & 9.9 & 3.8 & -6.1 & -3.0 & 2.1 & 5.1 & 11.0 & 5.0 & -6.0 \\
\hline Hoek van Holland & 23 & 81.6 & 54.7 & 10.7 & 5.9 & -4.7 & -4.8 & 0.8 & 5.5 & 12.9 & 6.0 & -6.8 \\
\hline Scheveningen & 24 & 78.2 & 66.1 & 5.9 & 2.3 & -3.6 & -5.3 & 0.5 & 5.8 & 9.6 & 2.5 & -7.1 \\
\hline IJmuiden buitenhaven & 25 & 69.9 & 96.7 & 2.7 & 1.4 & -1.3 & -5.7 & -0.3 & 5.4 & 7.6 & 1.4 & -6.1 \\
\hline K13a platform & 26 & 52.4 & 174.8 & 3.1 & 1.7 & -1.4 & -0.0 & -0.5 & -0.5 & 3.1 & 1.8 & -1.3 \\
\hline Petten zuid & 27 & 70.6 & 125.6 & -0.5 & -0.8 & -0.3 & -3.5 & 0.4 & 3.9 & 4.4 & 0.9 & -3.4 \\
\hline Terschelling Noordzee & 28 & 88.5 & 201.5 & 2.0 & -2.5 & -4.5 & -1.8 & 0.6 & 2.4 & 3.5 & 2.6 & -0.8 \\
\hline Wierumergronden & 29 & 94.1 & 228.6 & 1.7 & -3.8 & -5.5 & -3.2 & -0.2 & 3.0 & 5.5 & 3.8 & -1.6 \\
\hline Huibertgat & 30 & 101.2 & 245.8 & 0.8 & -5.1 & -5.9 & -5.6 & -2.3 & 3.3 & 9.9 & 6.5 & -3.5 \\
\hline Wilhelmshaven & 31 & 171.7 & 327.0 & -9.3 & -17.4 & -8.0 & -10.5 & -7.7 & 2.8 & 31.9 & 27.9 & -4.0 \\
\hline Cuxhaven & 32 & 135.3 & 339.2 & -4.3 & -9.5 & -5.2 & 1.1 & 4.2 & 3.1 & 5.0 & 13.5 & 8.5 \\
\hline Wittduen & 33 & 116.2 & 351.5 & 0.3 & -5.3 & -5.6 & -19.2 & -15.6 & 3.6 & 38.9 & 31.3 & -7.6 \\
\hline Tregde & 34 & 10.0 & 89.0 & 8.3 & 5.9 & -2.4 & -4.7 & -6.3 & -1.6 & 8.4 & 6.1 & -2.3 \\
\hline RMS & - & 134.5 & 194.1 & 9.9 & 5.2 & -4.7 & 5.4 & 4.2 & -1.2 & 16.6 & 11.7 & -4.9 \\
\hline Bias & - & - & - & 7.6 & -0.1 & -7.8 & -3.6 & -1.1 & 2.5 & - & - & - \\
\hline
\end{tabular}

Difference (SAL-no SAL) for each parameter is given by $\Delta$

Table 8 Impact of SAL effect in M2 amplitude error $(\Delta H)$, phase error $(\Delta G)$ and vector difference (VD) per station in the Dutch Coast

\begin{tabular}{|c|c|c|c|c|c|c|c|c|c|c|c|c|}
\hline \multirow[t]{2}{*}{ Station } & \multirow[t]{2}{*}{ St. no. } & \multirow[t]{2}{*}{$H_{\mathrm{o}}$} & \multirow[t]{2}{*}{$G_{\mathrm{o}}$} & \multicolumn{3}{|l|}{$\Delta H(\mathrm{~cm})$} & \multicolumn{3}{|l|}{$\Delta G\left({ }^{\circ}\right)$} & \multicolumn{3}{|l|}{$\mathrm{VD}(\mathrm{cm})$} \\
\hline & & & & No SAL & SAL & $\Delta$ & No SAL & SAL & $\Delta$ & No SAL & SAL & $\Delta$ \\
\hline Cadzand & 16 & 166.3 & 17.6 & 8.5 & -1.6 & -10.1 & -4.2 & -0.9 & 3.3 & 15.2 & 3.1 & -12.1 \\
\hline Westkapelle & 17 & 153.5 & 21.8 & 7.7 & -1.9 & -9.6 & -4.0 & -0.4 & 3.6 & 13.4 & 2.3 & -11.2 \\
\hline Roompot buiten & 19 & 136.1 & 32.5 & 4.0 & -4.0 & -7.9 & 0.7 & 4.5 & 3.7 & 4.3 & 11.2 & 6.9 \\
\hline Brouwershav. Gat 08 & 20 & 111.8 & 36.5 & 7.8 & 0.5 & -7.3 & -3.6 & 1.0 & 4.6 & 10.7 & 1.9 & -8.7 \\
\hline Haringvliet 10 & 21 & 96.0 & 42.0 & 10.8 & 4.5 & -6.3 & -4.5 & 0.5 & 5.0 & 13.4 & 4.6 & -8.8 \\
\hline Hoek van Holland & 23 & 81.6 & 54.7 & 10.7 & 5.9 & -4.7 & -4.8 & 0.8 & 5.5 & 12.9 & 6.0 & -6.8 \\
\hline Scheveningen & 24 & 78.2 & 66.1 & 5.9 & 2.3 & -3.6 & -5.3 & 0.5 & 5.8 & 9.6 & 2.5 & -7.1 \\
\hline IJmuiden buitenhaven & 25 & 69.9 & 96.7 & 2.7 & 1.4 & -1.3 & -5.7 & -0.3 & 5.4 & 7.6 & 1.4 & -6.1 \\
\hline Petten zuid & 27 & 70.6 & 125.6 & -0.5 & -0.8 & -0.3 & -3.5 & 0.4 & 3.9 & 4.4 & 0.9 & -3.4 \\
\hline K13a platform & 26 & 52.4 & 174.8 & 3.1 & 1.7 & -1.4 & -0.0 & -0.5 & -0.5 & 3.1 & 1.8 & -1.3 \\
\hline Terschelling Noordzee & 28 & 88.5 & 201.5 & 2.0 & -2.5 & -4.5 & -1.8 & 0.6 & 2.4 & 3.5 & 2.6 & -0.8 \\
\hline Wierumergronden & 29 & 94.1 & 228.6 & 1.7 & -3.8 & -5.5 & -3.2 & -0.2 & 3.0 & 5.5 & 3.8 & -1.6 \\
\hline Huibertgat & 30 & 101.2 & 245.8 & 0.8 & -5.1 & -5.9 & -5.6 & -2.3 & 3.3 & 9.9 & 6.5 & -3.5 \\
\hline RMS & - & 105.2 & 130.5 & 6.2 & 3.2 & -3.0 & 4.0 & 1.5 & -2.5 & 9.6 & 4.6 & -5.0 \\
\hline Bias & - & - & - & 5.0 & -0.2 & -5.3 & -3.5 & 0.3 & 3.8 & - & - & - \\
\hline
\end{tabular}

Difference (SAL-no SAL) for each parameter is given by $\Delta$ 


\section{References}

Accad Y, Pekeris CL (1978) Solution of the tidal equations for the M2 and S2 tides in the world oceans from a knowledge of the tidal potential alone. Philosophical Transactions of the Royal Society of London A: Mathematical, Physical and Engineering Sciences 290(1368): 235-266

Arbic BK, Garner ST, Hallberg RW, \& Simmons HL (2004) The accuracy of surface elevations in forward global barotropic and baroclinic tide models. Deep-Sea Res II Top Stud Oceanogr 51(25):3069-3101

Deltares (2016) Delft3D-FM user's manual 1.1.0 (Delft, The Netherlands)

Egbert GD, \& Erofeeva SY (2002) Efficient inverse modeling of barotropic ocean tides. J Atmos Ocean Technol 19(2):183-204

Egbert G D, Ray R D, \& Bills BG (2004) Numerical modeling of the global semidiurnal tide in the present day and in the last glacial maximum. J Geophys Res Oceans 109(C3)

Dziewonski AM, Anderson DL (1981) Preliminary reference Earth model. Phys Earth Planet Inter 25(4):297-356

Fang G, Xu X, Wei Z, Wang Y, Wang X (2013) Vertical displacement loading tides and self-attraction and loading tides in the Bohai, Yellow, and East China Seas. Science China Earth Sciences 56(1): 63-70

Farrell WE (1973) Earth tides, ocean tides and tidal loading. Philosophical Transactions of the Royal Society of London A: Mathematical, Physical and Engineering Sciences 274(1239):253259

Gordeev RG, Kagan BA, Polyakov EV (1977) The effects of loading and self-attraction on global ocean tides: the model and the results of a numerical experiment. J Phys Oceanogr 7(2):161-170

Hendershott MC (1972) The effects of solid earth deformation on global ocean tides. Geophys J Int 29(4):389-402

Jayne SR, St Laurent LC (2001) Parameterizing tidal dissipation over rough topography. Geophys Res Lett 28(5):811-814

Kernkamp HW, Van Dam A, Stelling GS, de Goede ED (2011) Efficient scheme for the shallow water equations on unstructured grids with application to the Continental Shelf. Ocean Dyn 61(8):1175-1188

Kuhlmann J, Dobslaw H, Thomas M (2011) Improved modeling of sea level patterns by incorporating self-attraction and loading. Journal of Geophysical Research: Oceans 116(C11)

Le Provost C, Genco ML, Lyard F (1995) Modeling and predicting tides over the World Ocean. Quantitative skill assessment for coastal ocean models 175-201

Le Provost C, Lyard F, Molines JM, Genco ML, Rabilloud F (1998) A hydrodynamic ocean tide model improved by assimilating a satellite altimeter-derived data set. Journal of Geophysical Research: Oceans 103(C3):5513-5529

Lyard F, Lefevre F, Letellier T, Francis O (2006) Modelling the global ocean tides: modern insights from FES2004. Ocean Dyn 56(5-6): 394-415
Maraldi C, Lyard F, Testut L, Coleman R (2011) Energetics of internal tides around the Kerguelen Plateau from modeling and altimetry. Journal of Geophysical Research: Oceans 116(C6)

Muis S, Verlaan M, Winsemius HC, Aerts JC, Ward PJ (2016) A global reanalysis of storm surges and extreme sea levels. Nature communications 7

Ngodock HE, Souopgui I, Wallcraft AJ, Richman JG, Shriver JF, Arbic BK (2016) On improving the accuracy of the M 2 barotropic tides embedded in a high-resolution global ocean circulation model. Ocean Model 97:16-26

Pawlowicz R, Beardsley B, Lentz S (2002) Classical tidal harmonic analysis including error estimates in MATLAB using T_TIDE. Comput Geosci 28(8):929-937

Ray RD (1998) Ocean self-attraction and loading in numerical tidal models. Mar Geod 21(3):181-192

Schrama E (2007) Tides. Lecture Notes AE4-876. http://www.deos. tudelft.n1/AS/ejo/ejo/ae4-876-tides.pdf

Shriver JF, Arbic BK, Richman JG, Ray RD, Metzger EJ, Wallcraft AJ, Timko PG (2012) An evaluation of the barotropic and internal tides in a high-resolution global ocean circulation model. Journal of Geophysical Research: Oceans 117(C10)

Stepanov VN, Hughes CW (2004) Parameterization of ocean selfattraction and loading in numerical models of the ocean circulation. Journal of Geophysical Research: Oceans 109(C3)

Tamisiea ME, Hill EM, Ponte RM, Davis JL, Velicogna I, Vinogradova NT (2010) Impact of self-attraction and loading on the annual cycle in sea level. Journal of Geophysical Research: Oceans 115(C7)

Teng F, Fang G, Xu X (2016) Effects of internal tidal dissipation and selfattraction and loading on semidiurnal tides in the Bohai Sea, Yellow Sea and East China Sea: a numerical study. Chinese Journal of Oceanology and Limnology 1-15

Verlaan M, De Kleermaeker S, Buckman L (2015) GLOSSIS: Global storm surge forecasting and information system. In Australasian Coasts \& Ports Conference 2015: 22nd Australasian Coastal and Ocean Engineering Conference and the 15th Australasian Port and Harbour Conference (p. 229). Engineers Australia and IPENZ

Vinogradova NT, Ponte RM, Tamisiea ME, Quinn KJ, Hill EM, Davis JL (2011) Self-attraction and loading effects on ocean mass redistribution at monthly and longer time scales. Journal of Geophysical Research: Oceans, 116(C8)

Wang H, Xiang L, Jia L, Jiang L, Wang Z, Hu B, Gao P (2012) Load Love numbers and Green's functions for elastic Earth models PREM, iasp91, ak135, and modified models with refined crustal structure from Crust 2.0. Comput Geosci 49:190-199

Zijl F, Verlaan M, Gerritsen H (2013) Improved water-level forecasting for the Northwest European Shelf and North Sea through direct modelling of tide, surge and non-linear interaction. Ocean Dyn 63(7):823-847

Zijl F, Sumihar J, Verlaan M (2015) Application of data assimilation for improved operational water level forecasting on the northwest European shelf and North Sea. Ocean Dyn 65(12):1699-1716 